\title{
Highly Enantioselective Michael Addition of Aromatic Ketones to Nitroolefins Promoted by Chiral Bifunctional Primary Amine-Thiourea Catalysts Based on Saccharides
}

\author{
Kun Liu, Han-Feng Cui, Jing Nie, Ke-Yan Dong, Xiao-Juan Li, Jun-An Ma* \\ Department of Chemistry, \\ Tianjin University, Tianjin 300072, China \\ Majun_an68@tju.edu.cn

\section{Supporting Information}

\section{Table of Contents}

Experimental Section
Copies of HPLC data
Copies of ${ }^{1} \mathrm{H}$ and ${ }^{13} \mathrm{C}$ NMR Spectra
Page 1 - Page 5

Page 6 - Page 19

Page 20 - Page 28

General information: ${ }^{1} \mathrm{H}$ and ${ }^{13} \mathrm{C}$ NMR were recorded on Brucker-300 and Varian-500 instruments. Chemical shifts were reported in ppm down field from internal $\mathrm{Me}_{4} \mathrm{Si}$. Optical rotations were determined using a Perkin Elmer Model 341 polarimeter. LRMS were recorded on a VG-7070E spectrometer. HRMS were done on a Bruker APEX III FTMS (7.0 TESLA). Chiral HPLC analyses were carried out on a Hewlett Packard Model HP 1200 instrument. All reactions were carried out in oven-dried glassware under air atmosphere unless otherwise noted.

Materials: All commercially available solvents and reagents were used as received unless otherwise noted. Analytical thin layer chromatography was performed on $0.20 \mathrm{~mm}$ Qingdao Haiyang silica gel plates. Silica gel (200-300 mesh) (from Qingdao Haiyang Chem. Company, Ltd.) was used for flash chromatography. Three saccharidyl isothiocyanates $\mathbf{1}$ were obtained following the literature procedure. ${ }^{[1]}$ 


\section{Preparation of catalysts:}

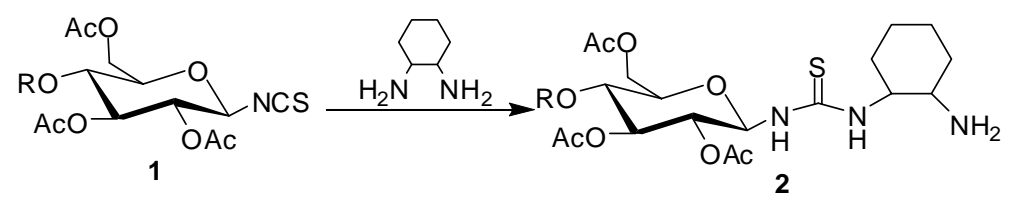

To a solution of 1, 2-cyclohexyldiamine $(3.6 \mathrm{mmol})$ in dichloromethane $(20 \mathrm{~mL})$ was added the corresponding sugar-derived isothiocyanates $1(3 \mathrm{mmol})$. The mixture was stirred at room temperature for 3-24 h (TLC) and concentrated. The resulting residue was chromatographed with the eluent (AcOEt / $\left.\mathrm{Et}_{3} \mathrm{~N}: 100 / 1\right)$ to give the crude solid. The crude solid was dissolved in a minimal amount of dichloromethane and slowly precipitated from solution by the addition of petroleum at $0{ }^{\circ} \mathrm{C}$. Filtration afforded the desired thiourea products 2 .

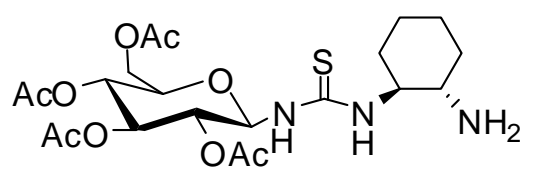

\section{$2 a$}

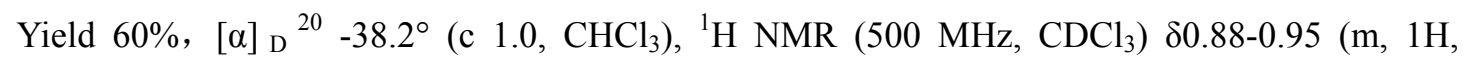
cyclohexane- $\underline{\mathrm{H}}), 1.24-1.27$ (m, 5H, cyclohexane- $\underline{\mathrm{H}}), 1.72-1.75$ (m, 2H, cyclohexane- $\underline{\mathrm{H}}), 2.00-2.09$

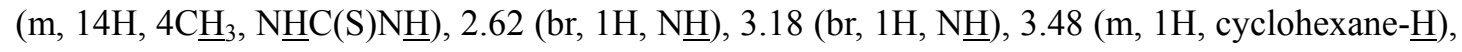
3.82-3.85 (m, 1H, cyclohexane- $\underline{\mathrm{H}}), 4.06-4.14\left(\mathrm{~m}, 2 \mathrm{H}, \underline{\mathrm{CH}}_{2}\right), 4.28-4.33(\mathrm{~m}, 1 \mathrm{H}$, pyranose- $\underline{\mathrm{H}})$, 5.03-5.11(m, 2H, pyranose- $\underline{\mathrm{H}}), 5.30-5.34(\mathrm{~m}, 1 \mathrm{H}$, pyranose- $\underline{\mathrm{H}}), 5.81-5.82(\mathrm{~m}, 1 \mathrm{H}$, pyranose- $\underline{\mathrm{H}})$; ${ }^{13} \mathrm{C}$ NMR $\left(125 \mathrm{MHz}, \mathrm{CDCl}_{3}\right) \delta 180.1,170.9,170.5,170.1,169.8,83.0,73.7,73.5,70.9,68.4,61.8$, 60.6, 53.6, 35.0, 32.1, 31.1, 24.7, 21.1, 21.0, 20.9, 20.8; IR (KBr): v 3349, 2929, 1752, 1542, 1373, 1229, 1035, 907, 758, $654 \mathrm{~cm}^{-1}$. MS (ESI): m/z $504.31\left(\mathrm{M}^{+}+1\right)$. HRMS (MALDI): Calculated for $\left[\mathrm{C}_{21} \mathrm{H}_{33} \mathrm{~N}_{3} \mathrm{O}_{9} \mathrm{~S}+\mathrm{H}\right]:$ 504.2025, found 504.2023.

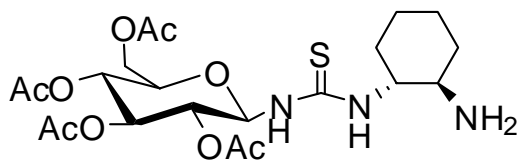

$2 b$

Yield $61 \%,[\alpha]_{\mathrm{D}}{ }^{20}+43.1^{\circ}\left(\mathrm{c} 1.0, \mathrm{CHCl}_{3}\right),{ }^{1} \mathrm{H}$ NMR $\left(500 \mathrm{MHz}, \mathrm{CDCl}_{3}\right) \delta 0.88-0.95(\mathrm{~m}, 1 \mathrm{H}$, cyclohexane- $\underline{\mathrm{H}}), 1.24-1.28$ (m, 5H, cyclohexane- $\underline{\mathrm{H}}), 1.72-1.74$ (m, 2H, cyclohexane- $\underline{\mathrm{H}}), 2.00-2.10$

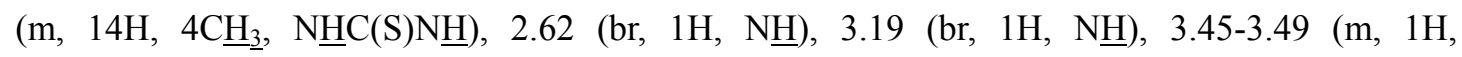
cyclohexane- $\underline{\mathrm{H}}), 3.82-3.85$ (m, 1H, cyclohexane- $\underline{\mathrm{H}}), 4.06-4.33$ (m, 3H, $\underline{\mathrm{H}}_{2}$, pyranose- $\left.\underline{\mathrm{H}}\right)$, 5.03-5.10(m, 2H, pyranose- $\underline{\mathrm{H}}), 5.30-5.34(\mathrm{~m}, 1 \mathrm{H}$, pyranose- $\underline{\mathrm{H}}), 5.81-5.83(\mathrm{~m}, 1 \mathrm{H}$, pyranose- $\underline{\mathrm{H}})$; ${ }^{13} \mathrm{C}$ NMR $\left(125 \mathrm{MHz}, \mathrm{CDCl}_{3}\right) \delta 179.1,170.9,170.5,170.1,169.8,83.0,73.7,73.5,70.9,68.4,61.8$, 
60.6, 53.6, 35.0, 32.1, 31.1, 24.7, 21.1, 21.0, 20.9, 20.8; IR (KBr): v 3349, 2929, 1752, 1542, 1373 , 1229, 1035, 907, 758, $654 \mathrm{~cm}^{-1}$. MS (ESI): m/z $504.30\left(\mathrm{M}^{+}+1\right)$. HRMS (MALDI): Calculated for $\left[\mathrm{C}_{21} \mathrm{H}_{33} \mathrm{~N}_{3} \mathrm{O}_{9} \mathrm{~S}+\mathrm{H}\right]:$ 504.2025, found 504.2024.

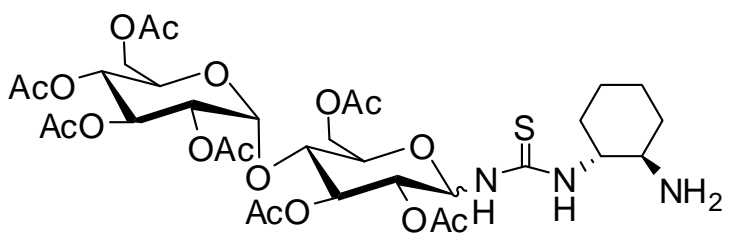

2c

Yield $60 \%,[\alpha]_{\mathrm{D}}{ }^{20}+103.6^{\circ}\left(\mathrm{c} 1.0, \mathrm{CHCl}_{3}\right),{ }^{1} \mathrm{H}$ NMR $\left(500 \mathrm{MHz}, \mathrm{CDCl}_{3}\right) \delta 1.24-1.27(\mathrm{~m}, 5 \mathrm{H}$, cyclohexane- $\underline{\mathrm{H}}), 1.73-1.80$ (m, 3H, cyclohexane- $\underline{\mathrm{H}}), 1.97-2.16$ (m, 23H, 7C$\left.\underline{H}_{3}, \underline{\mathrm{N}} \underline{\mathrm{HC}}(\mathrm{S}) \mathrm{N} \underline{\mathrm{H}}\right), 3.19$

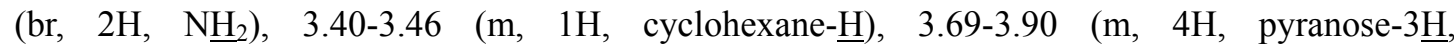
cyclohexane- $\underline{\mathrm{H}}), 4.05-4.22$ (m, 5H, pyranose- $\underline{\mathrm{H}}), 4.47-4.53$ (m, 1H, pyranose- $\underline{\mathrm{H}}), 4.66-4.74(\mathrm{~m}$, $1 \mathrm{H}$, pyranose- $\underline{\mathrm{H}}), 4.94-5.02(\mathrm{~m}, 1 \mathrm{H}$, pyranose- $\underline{\mathrm{H}}), 5.08-5.13(\mathrm{~m}, 1 \mathrm{H}$, pyranose- $\underline{\mathrm{H}}), 5.20-5.36(\mathrm{~m}$, $2 \mathrm{H}$, pyranose- $\underline{\mathrm{H}}) ;{ }^{13} \mathrm{C}$ NMR $\left(125 \mathrm{MHz}, \mathrm{CDCl}_{3}\right) \delta 184.2,171.3,171.0,170.5,170.3,170.2,169.7$, $169.2,101.1,88.4,76.4,73.2,71.9,71.2,69.3,68.5,66.9,66.8,63.9,61.0,60.5,56.4,33.7,30.8$, 25.1, 24.7, 21.3, 21.2, 21.1, 21.0, 20.9, 20.8, 20.6; IR (KBr): v 3359, 2931, 2858, 1741, 1541, 1234, 1050, $600 \mathrm{~cm}^{-1}$. MS (ESI): m/z $792.25\left(\mathrm{M}^{+}+1\right)(85 \%), 733.2(100 \%), 691.2(78 \%), 649.2$ (30\%). HRMS (MALDI): Calculated for $\left[\mathrm{C}_{33} \mathrm{H}_{49} \mathrm{~N}_{3} \mathrm{O}_{17} \mathrm{~S}\right]:$ : 791.2803, found 791.2792.

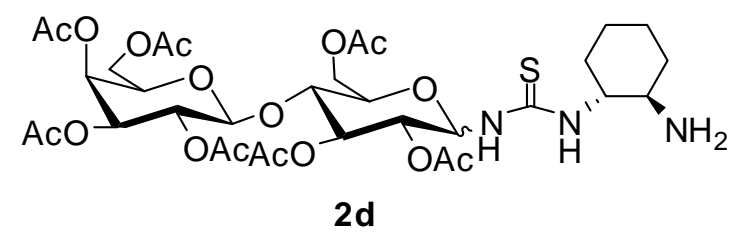

Yield $73 \%,[\alpha]_{\mathrm{D}}{ }^{20}+11.2^{\circ}\left(\mathrm{c} 1.0, \mathrm{CHCl}_{3}\right),{ }^{1} \mathrm{H}$ NMR $\left(500 \mathrm{MHz}, \mathrm{CDCl}_{3}\right) \delta 1.22-1.28(\mathrm{~m}, 5 \mathrm{H}$, cyclohexane- $\underline{\mathrm{H}}), 1.73-1.82$ (m, 3H, cyclohexane- $\underline{\mathrm{H}}), 1.96-2.16$ (m, 23H, 7C$\left.\underline{H}_{3}, \underline{\mathrm{N}} \underline{\mathrm{HC}}(\mathrm{S}) \mathrm{N} \underline{\mathrm{H}}\right), 3.19$ (br, $\left.2 \mathrm{H}, \quad \underline{\mathrm{H}}_{2}\right), \quad 3.40-3.47(\mathrm{~m}, \quad 1 \mathrm{H}, \quad$ cyclohexane- $\underline{\mathrm{H}}), \quad 3.48-3.90 \quad(\mathrm{~m}, 4 \mathrm{H}$, pyranose- $\underline{3 \mathrm{H}}$, cyclohexane- $\underline{\mathrm{H}}), 4.09-4.35$ (m, 5H, pyranose- $\underline{\mathrm{H}}), 4.42-4.60$ (m, 1H, pyranose- $\underline{\mathrm{H}}), 4.62-4.79(\mathrm{~m}$, $1 \mathrm{H}$, pyranose- $\underline{\mathrm{H}}), 4.93-5.09(\mathrm{~m}, 1 \mathrm{H}$, pyranose- $\underline{\mathrm{H}}), 5.10-5.13(\mathrm{~m}, 1 \mathrm{H}$, pyranose- $\underline{\mathrm{H}}), 5.26-5.36(\mathrm{~m}$ $2 \mathrm{H}$, pyranose- $\underline{\mathrm{H}}) ;{ }^{13} \mathrm{C}$ NMR $\left(125 \mathrm{MHz}, \mathrm{CDCl}_{3}\right) \delta 192.3,171.3,171.2,170.8,170.7,170.0,169.9$, $169.6,95.7,82.7,75.3,74.1,73.0,71.3,70.2,69.4,68.4,68.1,62.9,61.6,60.6,53.7,45.0,31.0$, 30.0, 29.2, 24.7, 27.1, 21.1, 21.0, 21.9, 20.8, 20.7; IR (KBr): v 3310, 2950, 1751, 1536, 1239, 
1031, $600 \mathrm{~cm}^{-1}$. MS (ESI): m/z $792.18\left(\mathrm{M}^{+}+1\right)(20 \%), 724.2(100 \%), 710.2(19 \%), 636.2(24 \%)$, 619.2 (22\%). HRMS (MALDI): Calculated for $\left[\mathrm{C}_{33} \mathrm{H}_{49} \mathrm{~N}_{3} \mathrm{O}_{17} \mathrm{~S}+\mathrm{H}\right]:$ : 792.2856, found 792.2856.

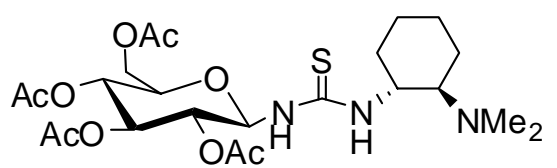

Yield $70 \%,[\alpha]_{\mathrm{D}}{ }^{20}+130.1^{\circ}\left(\mathrm{c} 1.0, \mathrm{CHCl}_{3}\right),{ }^{1} \mathrm{H}$ NMR $\left(500 \mathrm{MHz}, \mathrm{CDCl}_{3}\right) \delta 0.80-1.40(\mathrm{~m}, 2 \mathrm{H}$, cyclohexane- $\underline{\mathrm{H}}), 1.80-2.32$ (m, 24H, $6 \mathrm{C}_{3}$, cyclohexane- $\underline{\mathrm{H}} \times \mathrm{x}$ ), 4.07 (m, 1H, pyranose- $\left.\underline{\mathrm{H}}\right)$,

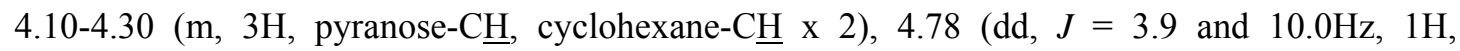
pyranose- $\underline{\mathrm{H}}), 4.91-5.27(\mathrm{~m}, 2 \mathrm{H}$, pyranose- $\underline{\mathrm{H}}), 5.48(\mathrm{t}, J=9.28 \mathrm{~Hz}, 1 \mathrm{H}$, pyranose- $\underline{\mathrm{H}}), 6.55(\mathrm{~d}, J=$ 4.0 Hz, $1 \mathrm{H}$, pyranose- $\underline{\mathrm{H}}) ;{ }^{13} \mathrm{C}$ NMR $\left(125 \mathrm{MHz}, \mathrm{CDCl}_{3}\right) \delta 180.8,170.6,170.0,169.8,169.5,88.8$, 74.3, 72.3, 70.7, 70.3, 67.3, 66.4, 61.1, 40.0, 20.81, 20.79, 20.76, 20.70; IR (KBr): v 3320, 2975, $2942,2780,1754,1541,1433,1372,1236,1112,1038,911,752,602 \mathrm{~cm}^{-1}$. MS (ESI): $\mathrm{m} / \mathrm{z} 532.20$ $\left(\mathrm{M}^{+}+1\right)$.

\section{Experimental Prodedures for Catalyzed Michael Addition of Aromatic Ketone to Nitroolefin:}

The nitroolefin $(0.3 \mathrm{mmol})$, aromatic ketone $(3.0 \mathrm{mmol})$ and catalyst $\mathbf{2 b}(0.045 \mathrm{mmol})$ were placed in a $5 \mathrm{~mL}$ vial equipped with a Teflon-coated stirbar. The solvent $(1 \mathrm{~mL})$ was added under air. The vial was capped with a white polyethylene stopper and the resulting mixture was stirred at room temperature for the stated time. Then the reaction solution was concentrated in vacuo and the crude was purified by flash chromatography to afford the product. Compounds $\mathbf{3 a},{ }^{[2,3,4]} \mathbf{3 b},{ }^{[3]} \mathbf{3} \mathbf{c},{ }^{[3,}$ ${ }^{4]} \mathbf{3} \mathbf{d},{ }^{[3]} \mathbf{3} \mathbf{e}^{[3]} \mathbf{3} \mathbf{f},{ }^{[3,5]} \mathbf{3 i},{ }^{[3]} \mathbf{3} \mathbf{j},{ }^{[3]} \mathbf{3} \mathbf{k}^{[2,3]}$ and $\mathbf{3} \mathbf{n}^{[3]}$ were known.

3g $[\alpha]_{\mathrm{D}}{ }^{20}-22.3^{\circ}$ (c 1.0, $\left.\mathrm{CHCl}_{3}\right),{ }^{1} \mathrm{H}$ NMR $\left(500 \mathrm{MHz}, \mathrm{CDCl}_{3}\right) \delta$ 7.94-7.92 (m, 1H, Ar-H), 7.85-7.74 (m, 4H, Ar-H), 7.59-7.40 (m, 7H, Ar-H), 4.92 (dd, J=4.8, 9.3 Hz, 1H, $\left.\mathrm{CH}_{2}\right), 4.78$ (dd, $J$ $\left.=6.0,9.6 \mathrm{~Hz}, 1 \mathrm{H}, \mathrm{CH}_{2}\right), 4.44-4.37(\mathrm{~m}, 1 \mathrm{H}, \mathrm{CH}), 3.55\left(\mathrm{dd}, J=5.1,9.6 \mathrm{~Hz}, 2 \mathrm{H}, \mathrm{CH}_{2}\right) .{ }^{13} \mathrm{C} \mathrm{NMR}$ $\left(75 \mathrm{MHz}, \mathrm{CDCl}_{3}\right) \delta 197.0,136.7,136.6,133.8,133.6,133.1,129.2,129.1128 .9,128.3,128.2$, 128.0, 127.9, 126.7, 126.6, 126.4, 125.3, 79.7, 41.8, 39.6; IR (KBr): v 3063, 2904, 1675, 1542, 1357, 1209, 748, $481 \mathrm{~cm}^{-1}$. MS (ESI): m/z $318.6\left(\mathrm{M}^{+}\right)(10 \%), 105$ (100\%), 77 (56\%), 51 (25\%). HRMS (MALDI): Calculated for $\left[\mathrm{C}_{20} \mathrm{H}_{17} \mathrm{NO}_{3}\right]$ : 319.1208 , found 319.1208 .

3h $[\alpha]_{\mathrm{D}}{ }^{20}-14.0^{\circ}\left(\mathrm{c} 1.0, \mathrm{CHCl}_{3}\right),{ }^{1} \mathrm{H}$ NMR $\left(300 \mathrm{MHz}, \mathrm{CDCl}_{3}\right) \delta$ 7.94(m, 2H), 7.60(m, 1H), 7.50(m, $2 \mathrm{H}), 7.34(\mathrm{~m}, 1 \mathrm{H}), 6.20(\mathrm{~d}, J=3 \mathrm{~Hz}, 2 \mathrm{H}), 4.77(\mathrm{~m}, 2 \mathrm{H}), 4.33(\mathrm{~m}, 1 \mathrm{H}), 3.48(\mathrm{~m}, 2 \mathrm{H}) ;{ }^{13} \mathrm{C} \mathrm{NMR}(75$ $\left.\mathrm{MHz}, \mathrm{CDCl}_{3}\right) \delta 196.8,152.2,142.5,136.5,133.9,129.0(2 \times \mathrm{C}), 128.3(2 \times \mathrm{C}), 110.7,107.4,77.6$, 
39.2, 33.4; IR (KBr): v 3063, 2914, 1675, 1557, 1388, 1199, 753, $599 \mathrm{~cm}^{-1}$. MS (ESI): $\mathrm{m} / \mathrm{z} 259.0$ $\left(\mathrm{M}^{+}\right)$.

$31[\alpha]_{\mathrm{D}}{ }^{20}-30.0^{\circ}\left(\mathrm{c} 0.1, \mathrm{CHCl}_{3}\right),{ }^{1} \mathrm{H}$ NMR $\left(500 \mathrm{MHz}, \mathrm{CDCl}_{3}\right) \delta 7.91(\mathrm{~d}, J=5.7 \mathrm{~Hz}, 1 \mathrm{H}, \mathrm{Ar}-\mathrm{H})$, 7.61-7.25 (m, 8H, Ar-H), 4.86-4.79 (m, 1H, $\left.\mathrm{CH}_{2}\right), 4.71-4.66\left(\mathrm{~m}, 1 \mathrm{H}, \mathrm{CH}_{2}\right), 4.25-4.18(\mathrm{~m}, 1 \mathrm{H}, \mathrm{CH})$, 3.47-3.39 (m, 2H, $\left.\mathrm{CH}_{2}\right) ;{ }^{13} \mathrm{C} \mathrm{NMR}\left(75 \mathrm{MHz}, \mathrm{CDCl}_{3}\right) \delta 197.0,151.0,139.3,136.6,133.8,132.3$, 129.7, $129.3(2 \times \mathrm{C}), 129.0,128.3,128.1,127.7,79.8,41.7,39.5$; IR (KBr): v 3062, 2924, 1685, 1574, 1383, 1234, 753, $692 \mathrm{~cm}^{-1}$. MS (ESI): m/z $346.8\left(\mathrm{M}^{+}-1\right)$ (45\%), $318.6(100 \%), 179.1(36 \%)$, $101.1(25 \%)$.

3m $[\alpha]_{\mathrm{D}}{ }^{20}-29.4^{\circ}\left(\mathrm{c} 1.0, \mathrm{CHCl}_{3}\right),{ }^{1} \mathrm{H}$ NMR $\left(500 \mathrm{MHz}, \mathrm{CDCl}_{3}\right) \delta 7.85(\mathrm{~d}, J=6.3 \mathrm{~Hz}, 1 \mathrm{H}, \mathrm{Ar}-\mathrm{H})$, 7.44-7.22 (m, 8H, Ar-H), 4.94-4.77 (m, 1H, $\left.\mathrm{CH}_{2}\right), 4.71-4.66\left(\mathrm{~m}, 1 \mathrm{H}, \mathrm{CH}_{2}\right), 4.22-4.17(\mathrm{~m}, 1 \mathrm{H}, \mathrm{CH})$, 3.45-3.41 (m, 2H, $\left.\left.\mathrm{CH}_{2}\right) ;{ }^{13} \mathrm{C} \mathrm{NMR} \mathrm{(75} \mathrm{MHz,} \mathrm{CDCl}_{3}\right) \delta 195.8,140.3,139.1,134.9,132.3,130.8$, 129.6, 129.3, 129.2, 128.2, 127.8, 127.6, 127.2, 79.7, 41.7, 39.5; IR (KBr): v 3088, 3017, 2909 , 1680, 1552, 1378, 1204, 978, 692, $564 \mathrm{~cm}^{-1}$. MS (ESI): m/z $303.2\left(\mathrm{M}^{+}\right)(100 \%), 243.1(85 \%)$, $225.3(20 \%)$.

\section{Optical rotation and chiral-phase HPLC data}

\begin{tabular}{|c|c|c|}
\hline Product & {$[\alpha]_{\mathrm{D}}^{20}$} & Daicel Chiralpak (AD-H) \\
\hline 3a & $-23.0^{\circ}\left(\mathrm{c} 1.0, \mathrm{CHCl}_{3}\right)$ & $i-\mathrm{PrOH} /$ hexane: $10 / 90,254 \mathrm{~nm}, 1.0 \mathrm{~mL} / \mathrm{min}, \mathrm{t}_{\mathrm{R}}=16.4 \mathrm{~min}$ (major), $23.2 \mathrm{~min}$ \\
\hline $3 \mathbf{b}$ & $-22.0^{\circ}\left(\mathrm{c} 1.0, \mathrm{CHCl}_{3}\right)$ & $i-\mathrm{PrOH} /$ hexane: $20 / 80,254 \mathrm{~nm}, 1.0 \mathrm{~mL} / \mathrm{min}, \mathrm{t}_{\mathrm{R}}=9.3 \mathrm{~min}$ (major), $12.2 \mathrm{~min}$ \\
\hline $3 \mathbf{c}$ & $-21.1^{\circ}\left(\mathrm{c} 1.0, \mathrm{CHCl}_{3}\right)$ & $i-\mathrm{PrOH} /$ hexane: $20 / 80,254 \mathrm{~nm}, 1.0 \mathrm{~mL} / \mathrm{min}, \mathrm{t}_{\mathrm{R}}=13.8 \mathrm{~min}$ (major), $18.9 \mathrm{~min}$ \\
\hline 3d & $-23.3^{\circ}\left(\mathrm{c} 1.0, \mathrm{CHCl}_{3}\right)$ & $i-\mathrm{PrOH} /$ hexane: $10 / 90,254 \mathrm{~nm}, 1.0 \mathrm{~mL} / \mathrm{min}, \mathrm{t}_{\mathrm{R}}=17.6 \mathrm{~min}$ (major), $25.9 \mathrm{~min}$ \\
\hline $3 e$ & $-26.1^{\circ}\left(\mathrm{c} 1.0, \mathrm{CHCl}_{3}\right)$ & $i$-PrOH/hexane: $15 / 85,254 \mathrm{~nm}, 1.0 \mathrm{~mL} / \mathrm{min}, \mathrm{t}_{\mathrm{R}}=12.0 \mathrm{~min}$ (major), $14.5 \mathrm{~min}$ \\
\hline 3f & $-29.4^{\circ}\left(\mathrm{c} 1.0, \mathrm{CHCl}_{3}\right)$ & $i-\mathrm{PrOH} /$ hexane: $15 / 85,254 \mathrm{~nm}, 1.0 \mathrm{~mL} / \mathrm{min}, \mathrm{t}_{\mathrm{R}}=11.1 \mathrm{~min}$ (major), $13.4 \mathrm{~min}$ \\
\hline $3 g$ & $-22.3^{\circ}\left(\mathrm{c} 1.0, \mathrm{CHCl}_{3}\right)$ & $i-\mathrm{PrOH} / \mathrm{hexane}: 10 / 90,254 \mathrm{~nm}, 1.0 \mathrm{~mL} / \mathrm{min}, \mathrm{t}_{\mathrm{R}}=23.3 \mathrm{~min}$ (major), $29.0 \mathrm{~min}$ \\
\hline $3 \mathbf{h}$ & $-14.0^{\circ}\left(\mathrm{c} 1.0, \mathrm{CHCl}_{3}\right)$ & $i-\mathrm{PrOH} /$ hexane: $10 / 90,254 \mathrm{~nm}, 1.0 \mathrm{~mL} / \mathrm{min}, \mathrm{t}_{\mathrm{R}}=12.1 \mathrm{~min}$ (major), $14.7 \mathrm{~min}$ \\
\hline $3 \mathbf{i}$ & $-2.4^{\circ}\left(\mathrm{c} 0.8, \mathrm{CHCl}_{3}\right)$ & $i-\mathrm{PrOH} /$ hexane: $10 / 90,254 \mathrm{~nm}, 1.0 \mathrm{~mL} / \mathrm{min}, \mathrm{t}_{\mathrm{R}}=6.7 \mathrm{~min}$ (major), $7.3 \mathrm{~min}$ \\
\hline $3 \mathbf{j}$ & $-32.1^{\circ}\left(\mathrm{c} 1.0, \mathrm{CHCl}_{3}\right)$ & $i-\mathrm{PrOH} /$ hexane: $10 / 90,254 \mathrm{~nm}, 1.0 \mathrm{~mL} / \mathrm{min}, \mathrm{t}_{\mathrm{R}}=18.5 \mathrm{~min}$ (major), $30.7 \mathrm{~min}$ \\
\hline $3 \mathbf{k}$ & $-28.4^{\circ}\left(\mathrm{c} 1.0, \mathrm{CHCl}_{3}\right)$ & $i-\mathrm{PrOH} /$ hexane: $15 / 85,254 \mathrm{~nm}, 1.0 \mathrm{~mL} / \mathrm{min}, \mathrm{t}_{\mathrm{R}}=24.3 \mathrm{~min}$ (major), $39.7 \mathrm{~min}$ \\
\hline \multirow[t]{2}{*}{31} & $-30.0^{\circ}\left(\mathrm{c} 0.1, \mathrm{CHCl}_{3}\right)$ & $i-\mathrm{PrOH} /$ hexane: $15 / 85,254 \mathrm{~nm}, 1.0 \mathrm{~mL} / \mathrm{min}, \mathrm{t}_{\mathrm{R}}=12.2 \mathrm{~min}$ (major), $16.6 \mathrm{~min}$ \\
\hline & & $18.9 \mathrm{~min}$ (major), $24.8 \mathrm{~min}$ \\
\hline $3 \mathrm{~m}$ & $-29.4^{\circ}\left(\mathrm{c} 1.0, \mathrm{CHCl}_{3}\right)$ & $i-\mathrm{PrOH} /$ hexane: $15 / 85,254 \mathrm{~nm}, 1.0 \mathrm{~mL} / \mathrm{min}, \mathrm{t}_{\mathrm{R}}=16.7 \mathrm{~min}$ (major), $22.2 \mathrm{~min}$ \\
\hline $3 n$ & $-58.7^{\circ}\left(\mathrm{c} 0.8, \mathrm{CHCl}_{3}\right)$ & $i-\mathrm{PrOH} /$ hexane: $10 / 90,254 \mathrm{~nm}, 1.0 \mathrm{~mL} / \mathrm{min}, \mathrm{t}_{\mathrm{R}}=25.7 \mathrm{~min}$ (major), $49.7 \mathrm{~min}$ \\
\hline
\end{tabular}

[1] a) Lindhorst, T.; Kieburg, C. Synthesis 1995, 10, 1228; b) Selkti, M.; Kassab, R.; Lopez, H. P.; Villain, F.; de Rango, C. J. Carbohydr. Chem. 1999, 18, 1019; c) Pearson, M. S. M.; Robin, A.; Bourgougnon, N.; Meslin, J. C.; Deniaud, D. J. Org. Chem. 2003, 68, 8583.

[2] Vakylya, B.; Varga, S.; Csapmpai, A.; Soós, T. Org. Lett. 2005, 7, 1967.

[3] Dixon, D. J.; Richardson, R. D.; Synlett. 2006, 1, 81.

[4] Huang, H.; Jacobsen, E. N. J. Am. Chem. Soc. 2006, 128, 7170.

[5] Corey, E. J.; Zhang, F.-Y. Org. Lett. 2000, 2, 4257. 


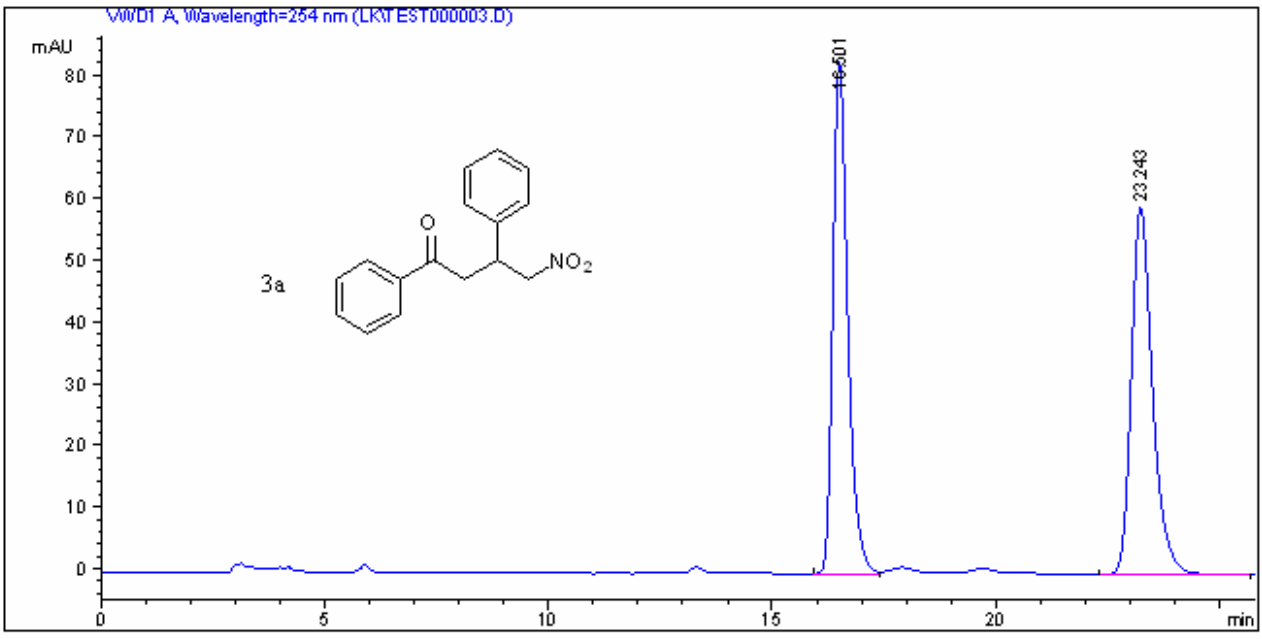

Area Percent Report

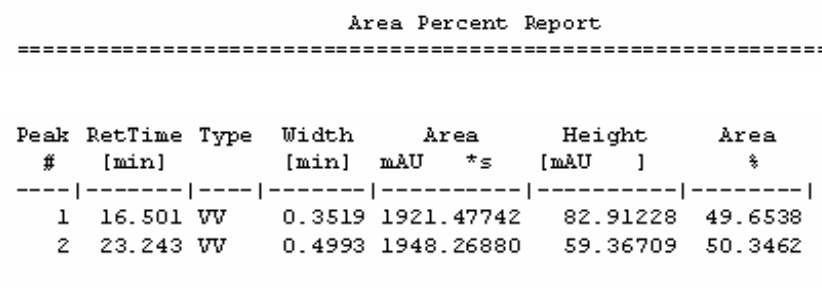

Totals:

$3869.74622 \quad 142.27937$

Sample Info : 254nm,i-ProH: hexane $=10: 90, \mathrm{AD}-\mathrm{H}, 1.0 \mathrm{ml} / \mathrm{min}$

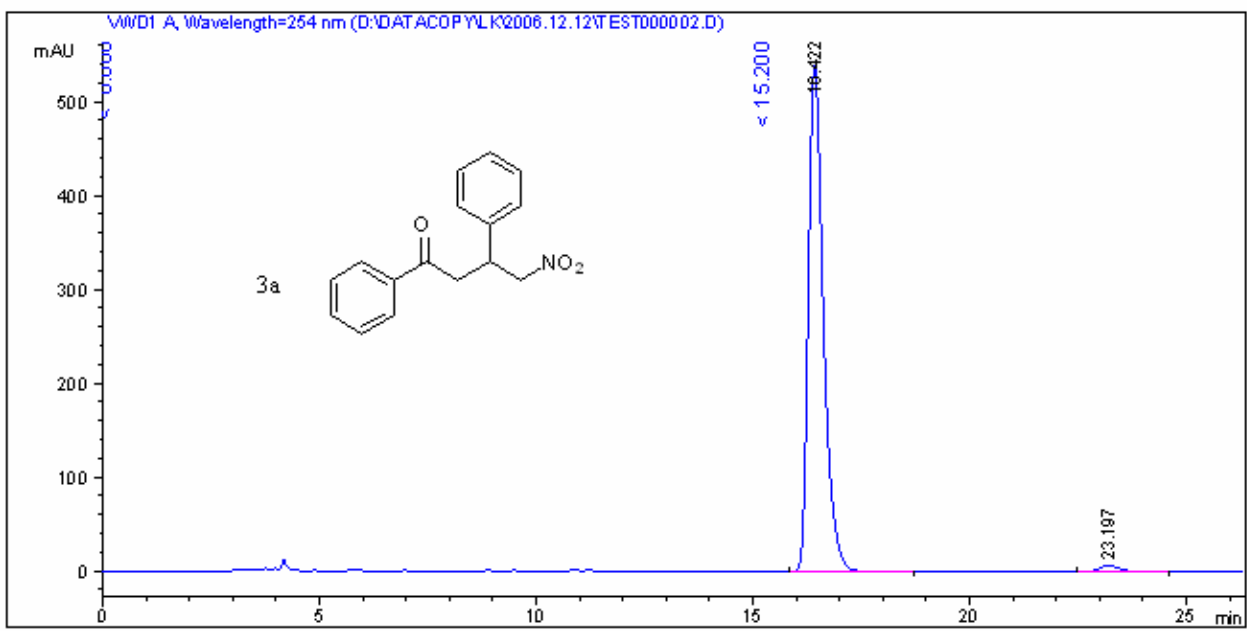

Area Percent Report
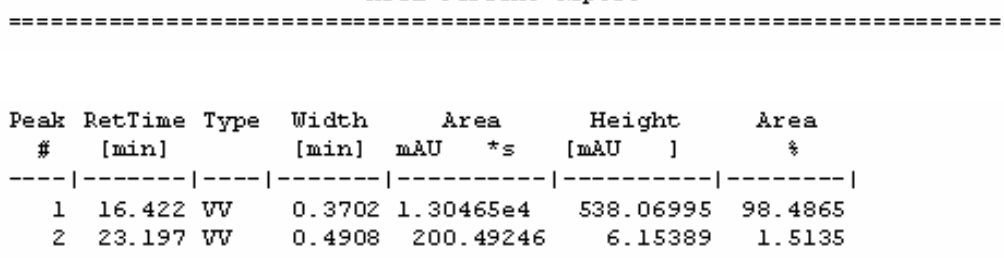

Totals :

1. $32470 e 4 \quad 544.22384$ 
Sample Info : $254 \mathrm{~nm}, \mathrm{i}-\mathrm{pr} 0 \mathrm{H}$ : hexane $=20: 80, \mathrm{AD}-\mathrm{H}, 1.0 \mathrm{ml} / \mathrm{min}$

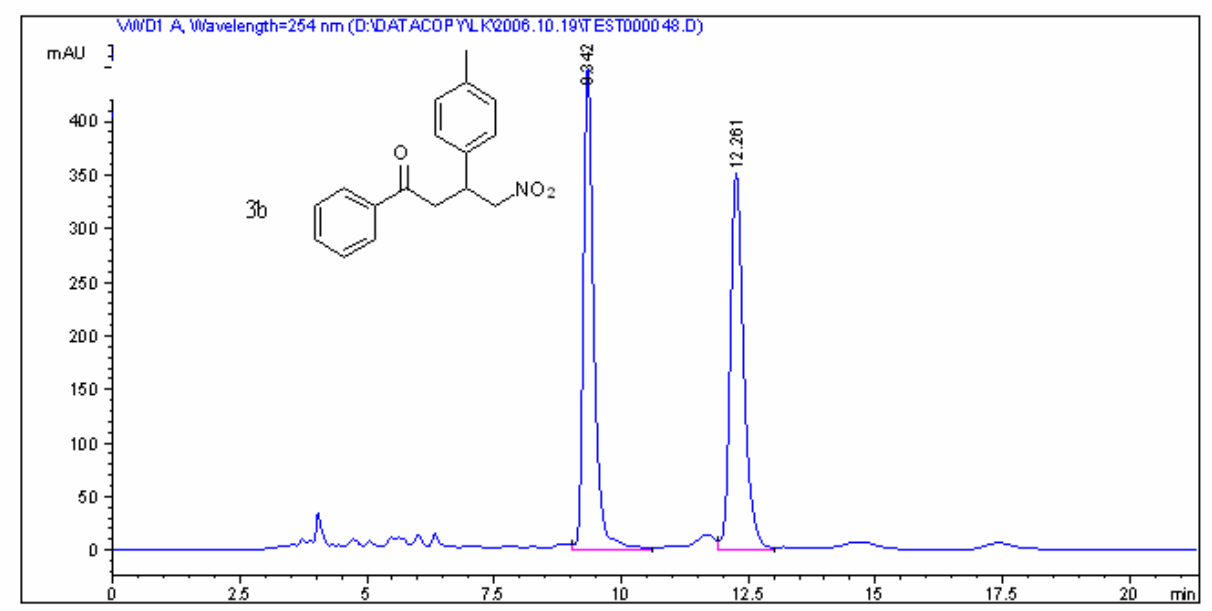

Area Percent Report

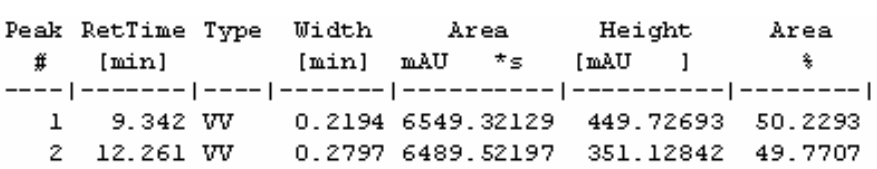

Totals :

1. 30388 e4 800.85535

Sample Info : $254 \mathrm{~nm}, \mathrm{i}-\mathrm{Pr} 0 \mathrm{H}:$ hexane $=20: 80, \mathrm{AD}-\mathrm{H}, 1.0 \mathrm{ml} / \mathrm{min}$

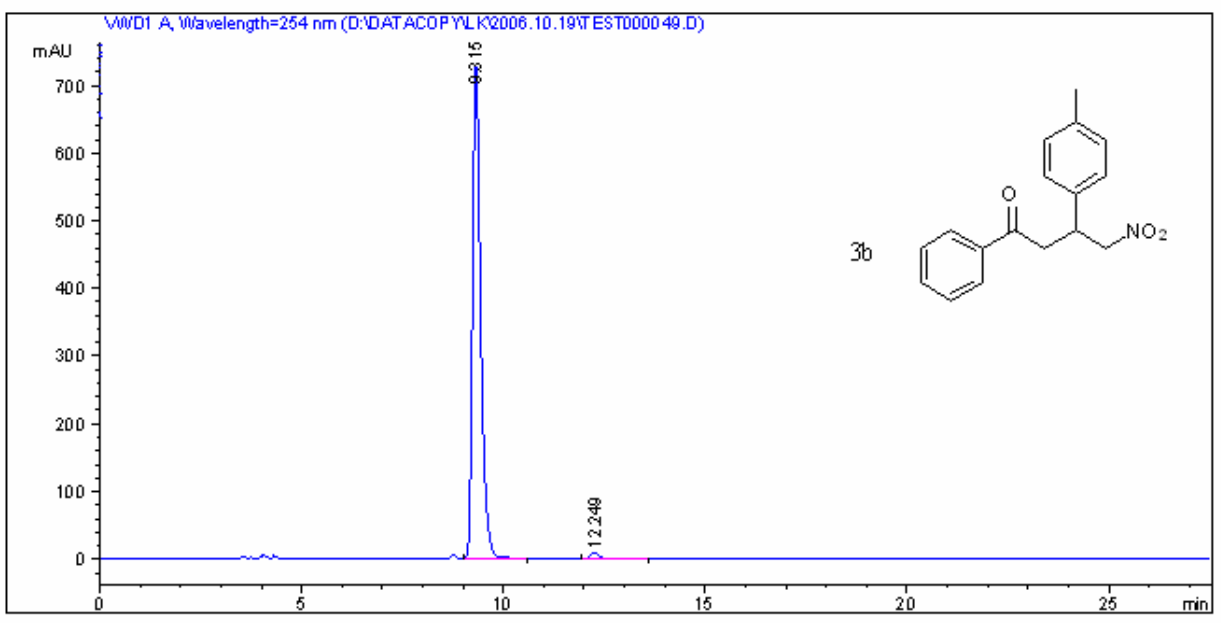

Area Percent Report

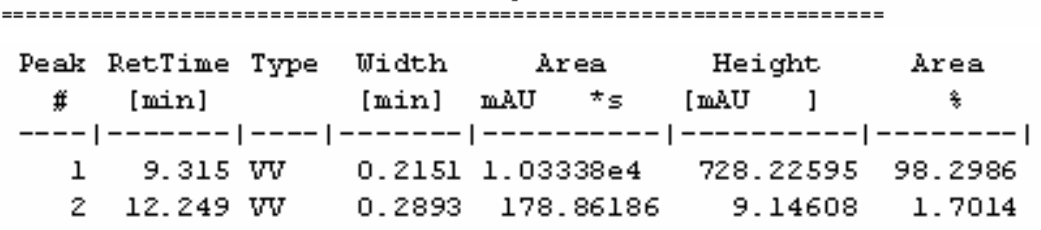

Totals :

1. $05126 e 4$

737.37204 


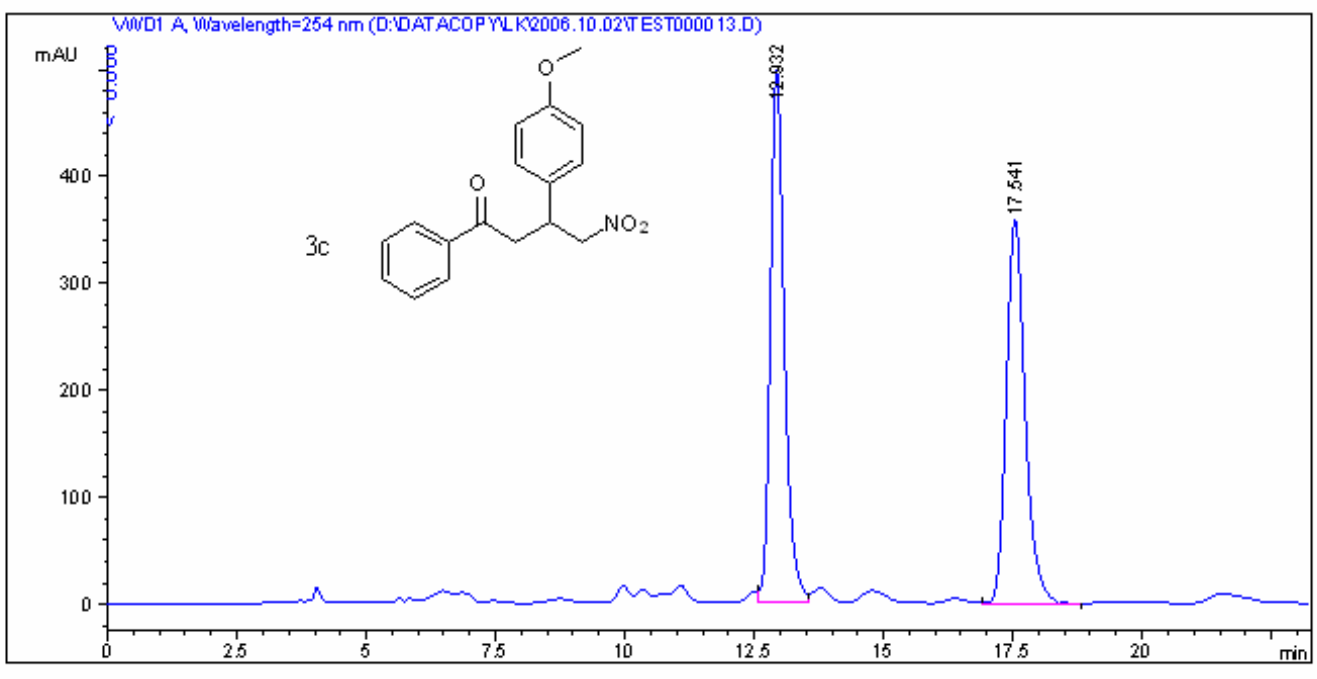

Area Percent Report

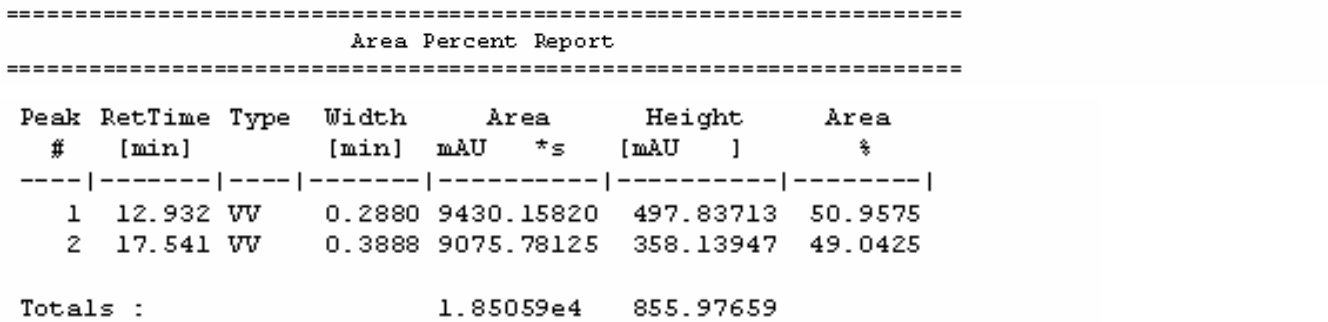

Sample Info : $254 \mathrm{~nm}, \mathrm{i}-\mathrm{Pr} 0 \mathrm{H}:$ hexane $=20: 80, \mathrm{AD}-\mathrm{H}, 1.0 \mathrm{ml} / \mathrm{min}$
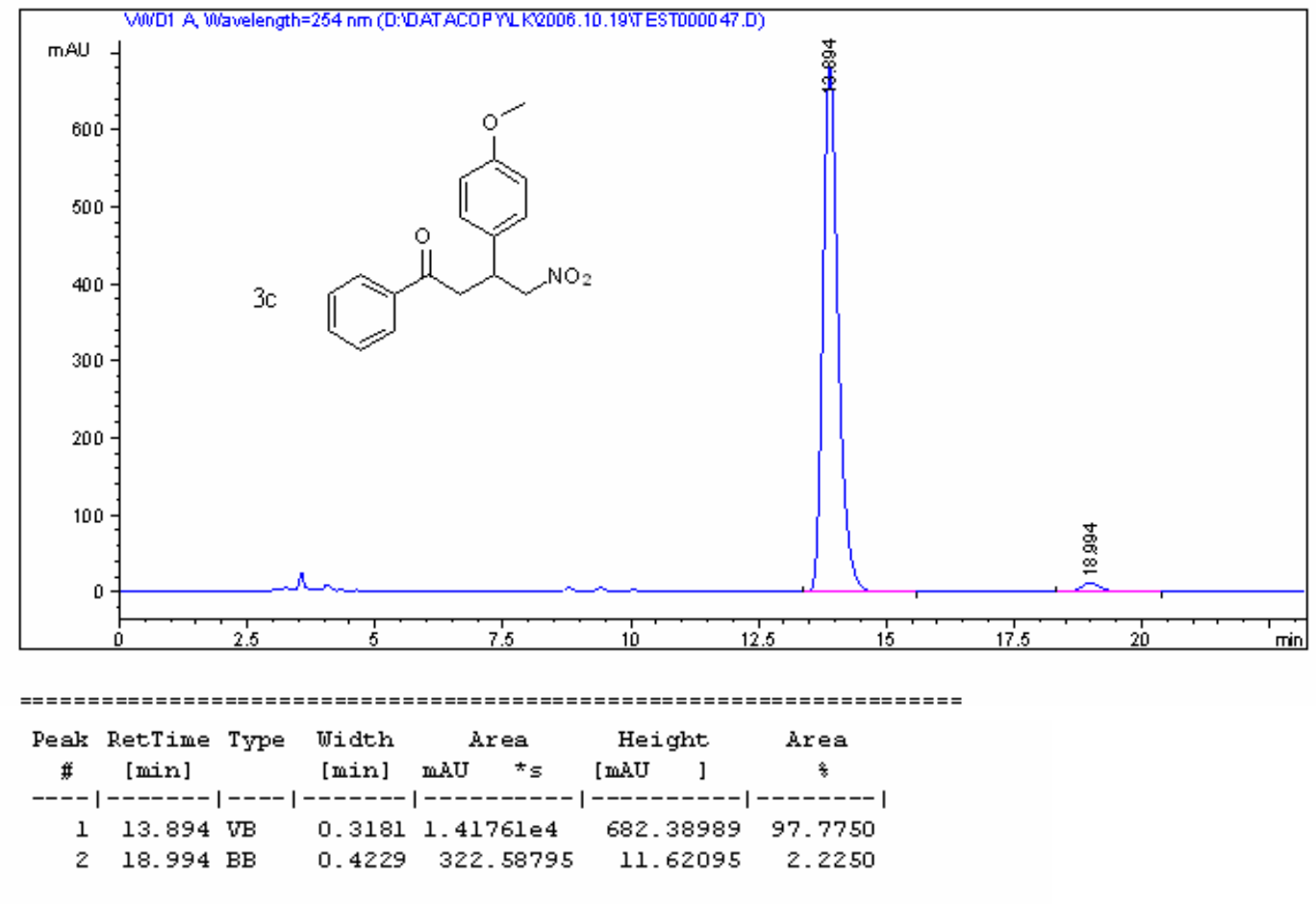

Totals: $\quad 1.44987 e 4 \quad 694.01084$ 
Sample Info : 254m, i-ProH: he xane $=10: 90$, AD-H, 1.0ml/min

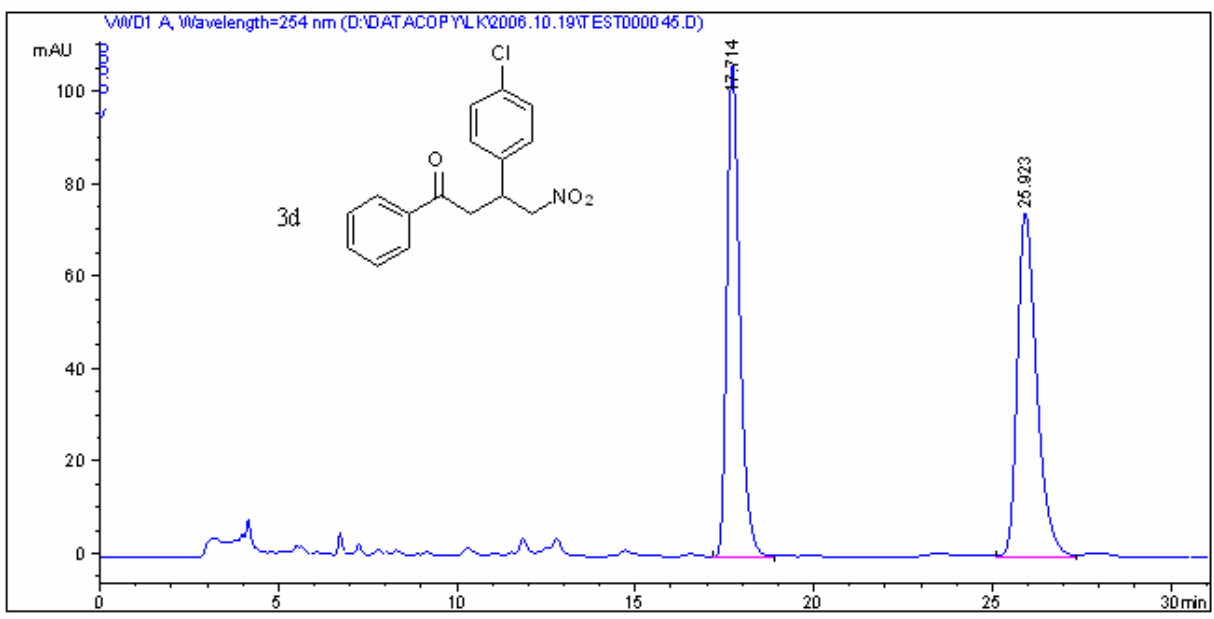

Area Percent Report

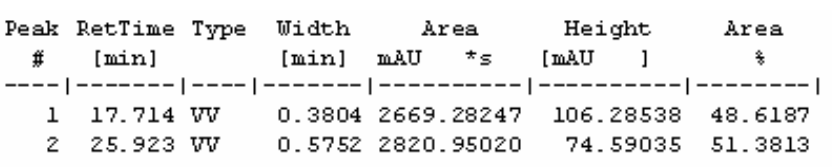

Totals :

$5490.23267 \quad 180.87573$

Sample Info : $254 \mathrm{~nm}, \mathrm{i}-\mathrm{PrOH}:$ hexane $=10: 90, \mathrm{AD}-\mathrm{H}, 1.0 \mathrm{ml} / \mathrm{min}$

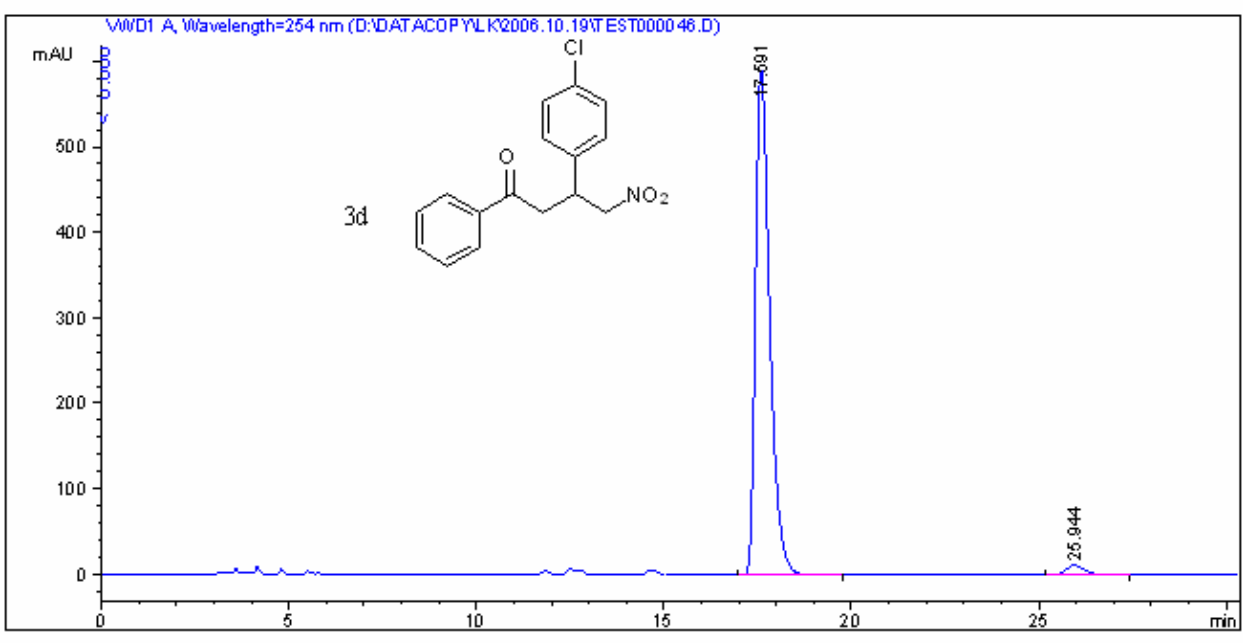

Area Percent Report

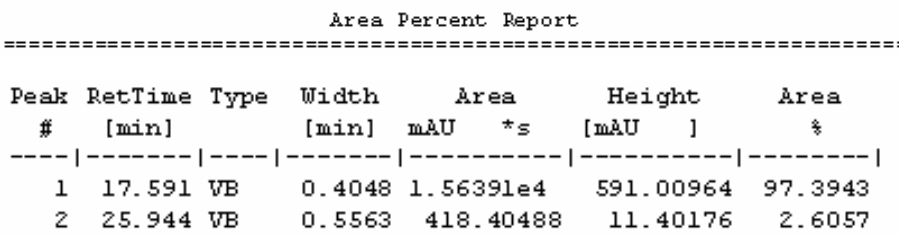

Totals :

$1.60575 \mathrm{e} 4602.41140$ 


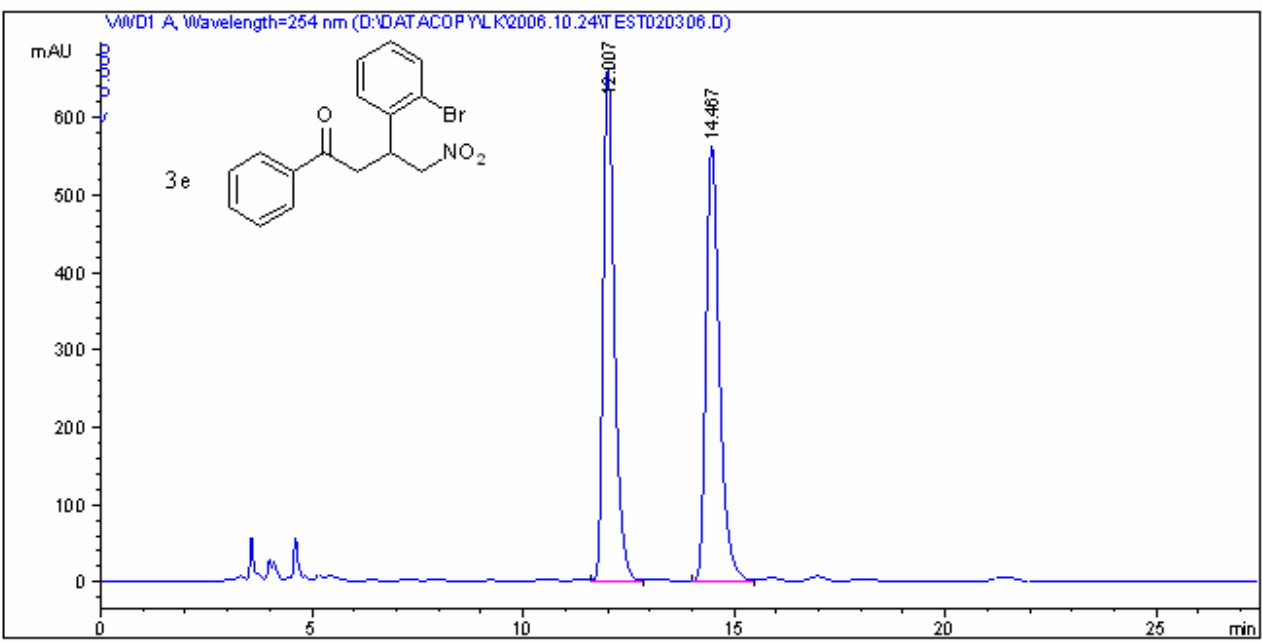

Area Percent Report

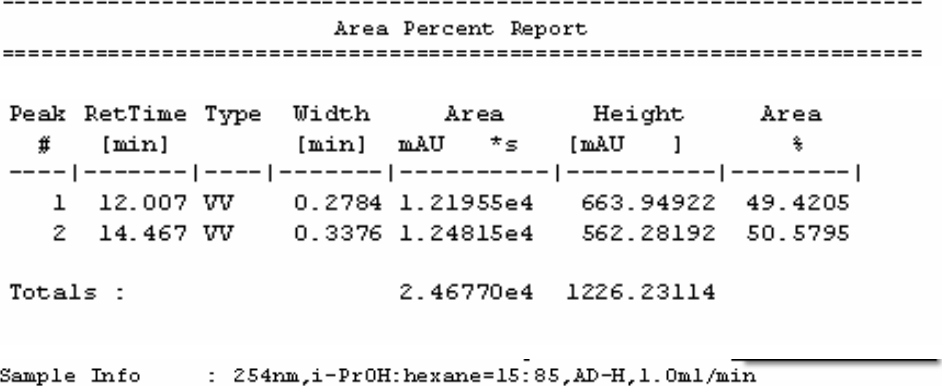

Sample Info : 254nm,i-ProH: hexane $=15: 85, \mathrm{AD}-\mathrm{H}, 1.0 \mathrm{ml} / \mathrm{min}$

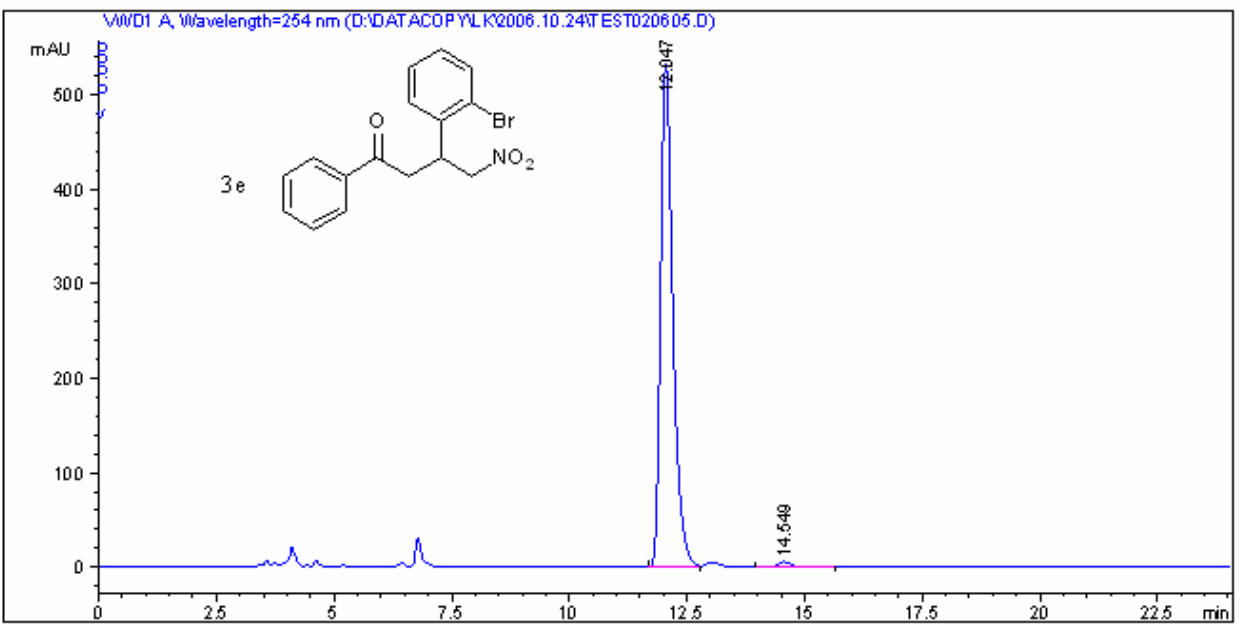

Area Percent Report

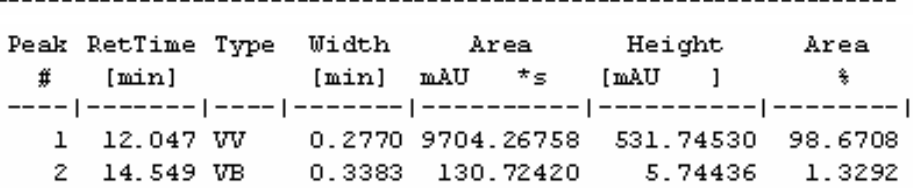

Totals :

$9834.99178 \quad 537.48966$ 
Sample Info : $254 \mathrm{~nm}, \mathrm{i}-\mathrm{Pr} 0 \mathrm{H}:$ hexane $=15: 85, \mathrm{AD}-\mathrm{H}, 1 \mathrm{ml} / \mathrm{min}$

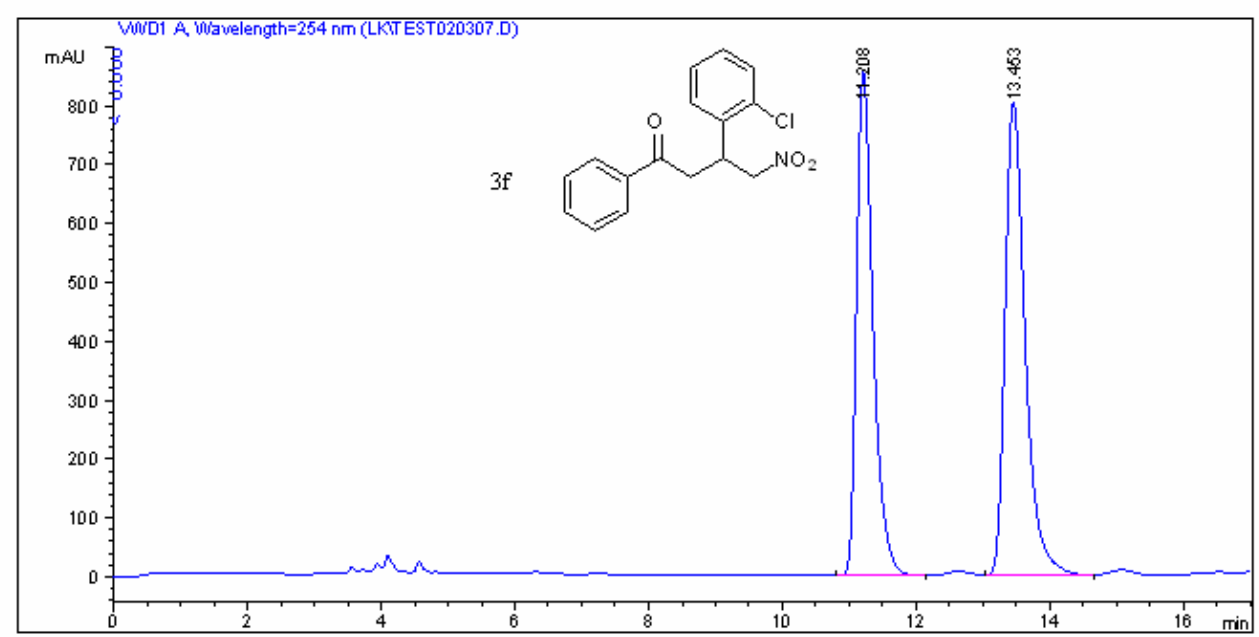

Area Percent Report

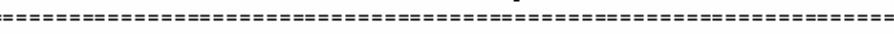

Peal RetTime Type width Area Height Area

\# [min]

----|-------|----|-------|----------|----------1--------

$\begin{array}{lllllll}1 & 11.208 & \text { VV } & 0.2621 & 1.47276 \mathrm{e} & 855.03442 & 46.6272\end{array}$

$2 \quad 13.453$ vV $0.32001 .68582 \mathrm{e} 4 \quad 804.95410 \quad 53.3728$

Totals :

3. 15858 e4 1659.98853

Sample Info : 254nm,i-ProH:hexane $=15: 85,4 \mathrm{D}-\mathrm{H}, 1 \mathrm{ml} / \mathrm{min}$

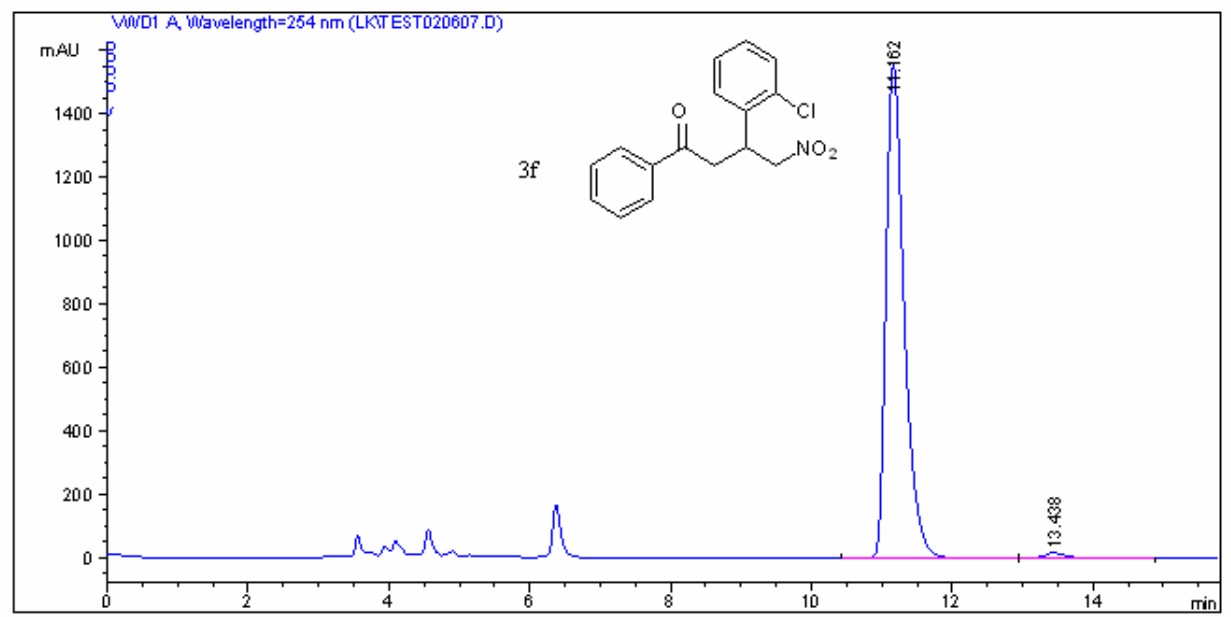

Area Percent Report

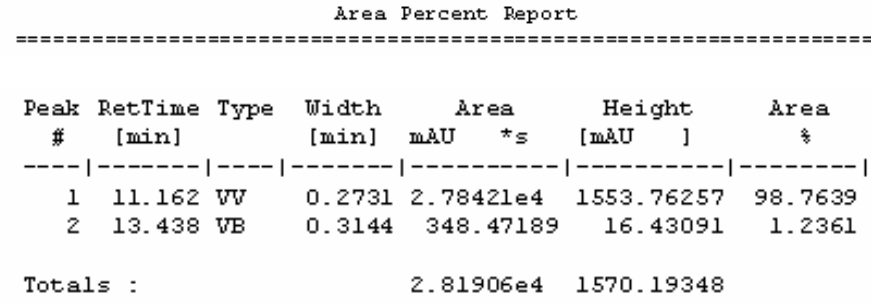

Page 11 of 28 
Sample Info : $254 \mathrm{~nm}, \mathrm{i}-\mathrm{Pr} 0 \mathrm{H}:$ he $\mathrm{xane}=10: 90, \mathrm{AD}-\mathrm{H}, 1.0 \mathrm{ml} / \mathrm{min}$

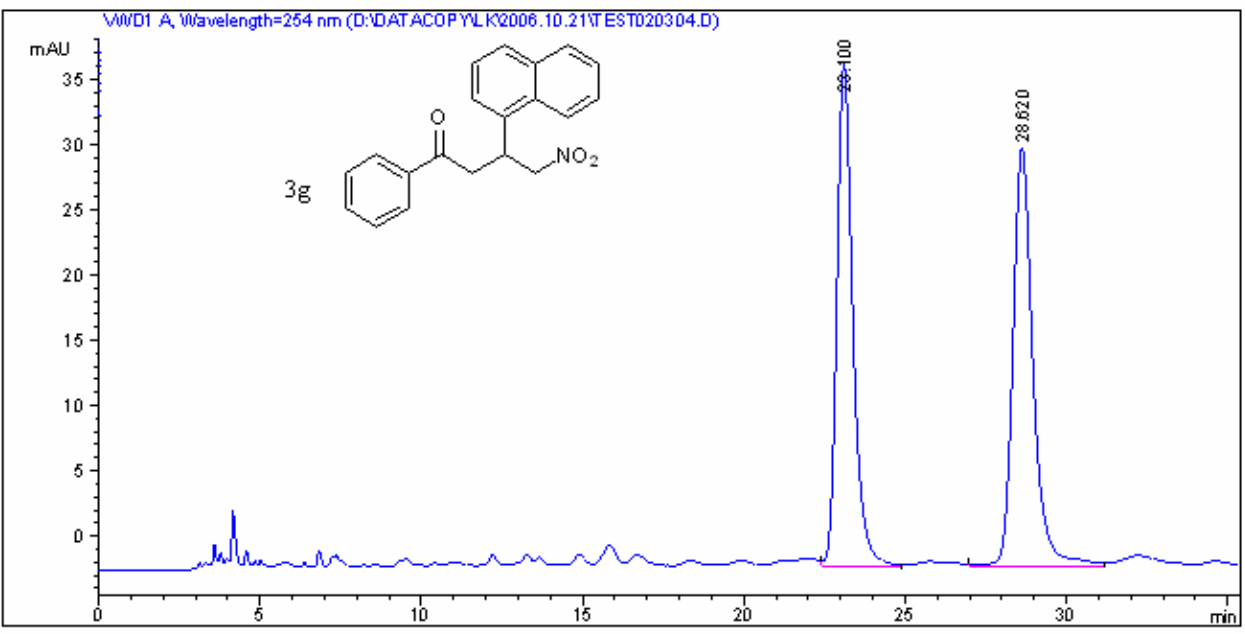

Area Percent Report
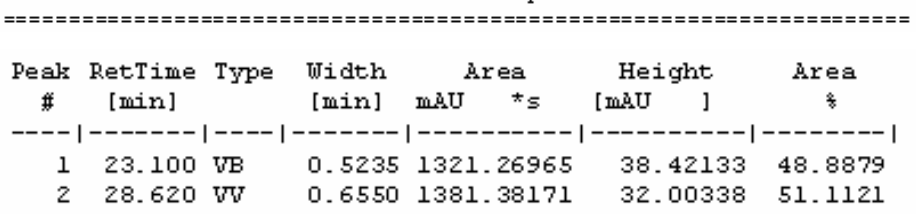

Totals :

$2702.65137 \quad 70.42471$

Sample Info : 254nm,i-ProH: hexane $=10: 90, \mathrm{AD}-\mathrm{H}, 1.0 \mathrm{ml} / \mathrm{min}$

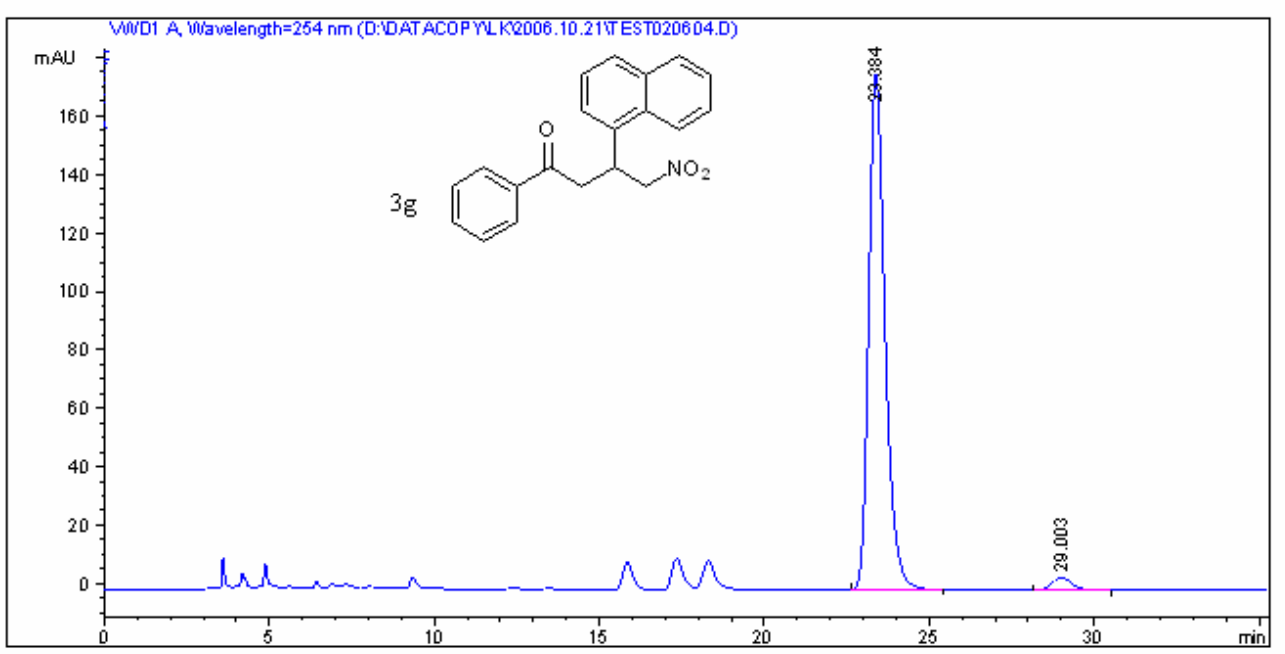

Area Percent Report

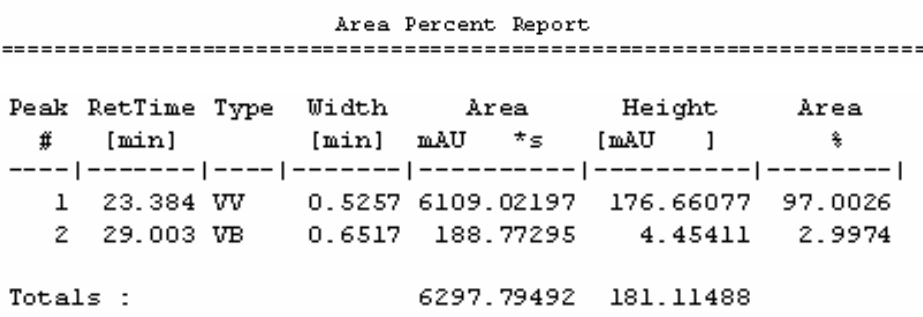

Page 12 of 28 
Sample Info : $254 \mathrm{~mm}, \mathrm{i}-\operatorname{Pr} 0 \mathrm{H}:$ hexane $=10: 90, \mathrm{AD}-\mathrm{H}, 1.0 \mathrm{ml} / \mathrm{min}$

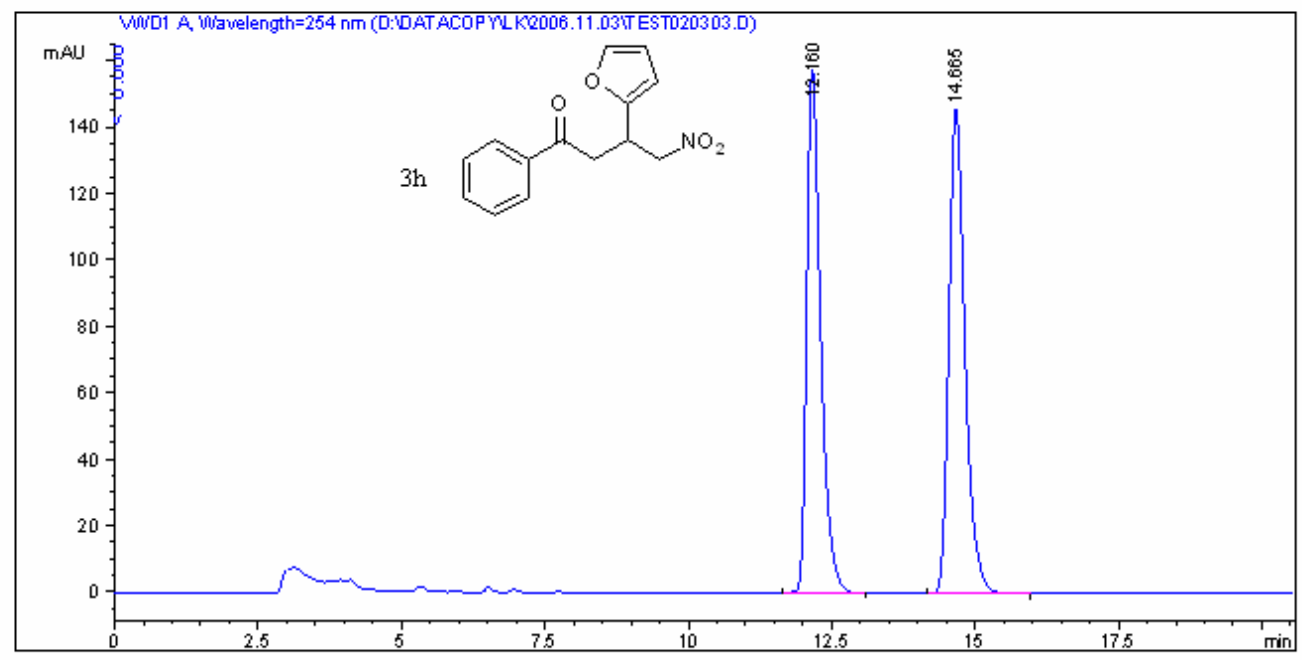

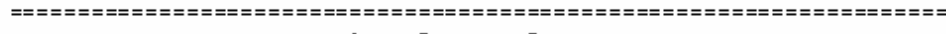

Area Percent Report

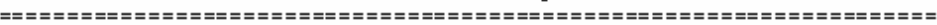

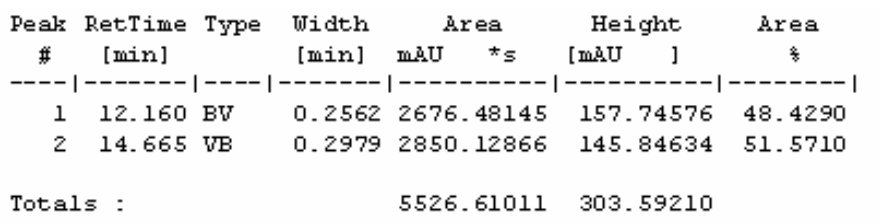

Sample Info : $254 \mathrm{~nm}, \mathrm{i}-\mathrm{PrOH}:$ hexane $=10: 90, \mathrm{AD}-\mathrm{H}, 1.0 \mathrm{ml} / \mathrm{min}$

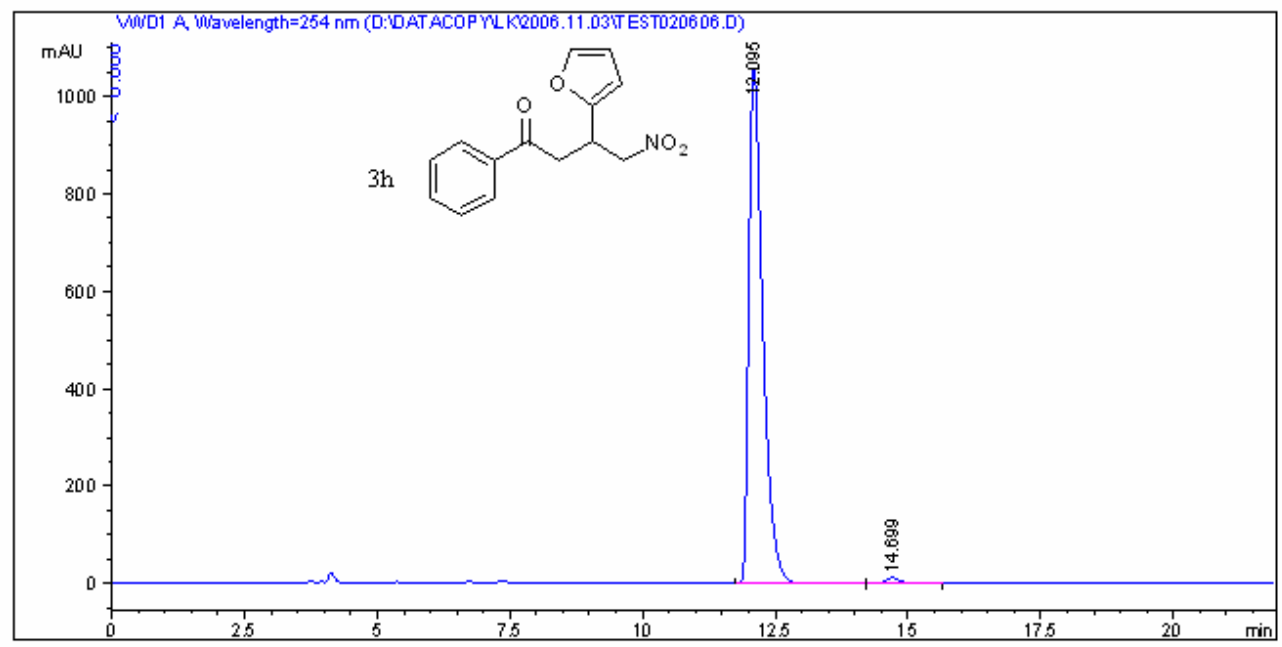

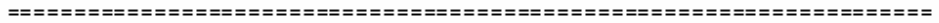

Area Percent Report

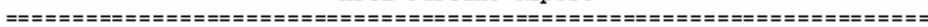

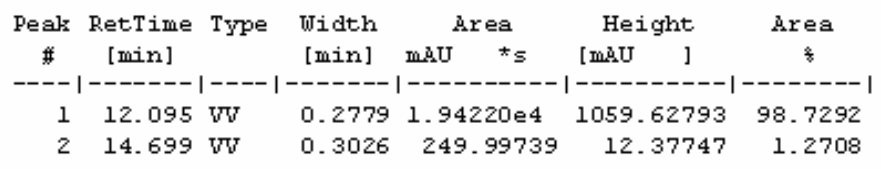

Totals :

$1.96720 e 4 \quad 1072.00540$

Page 13 of 28 
Sample Info : 254m,i-ProH: hexane $=10: 90, \mathrm{AD}-\mathrm{H}, 1.0 \mathrm{ml} / \mathrm{min}$
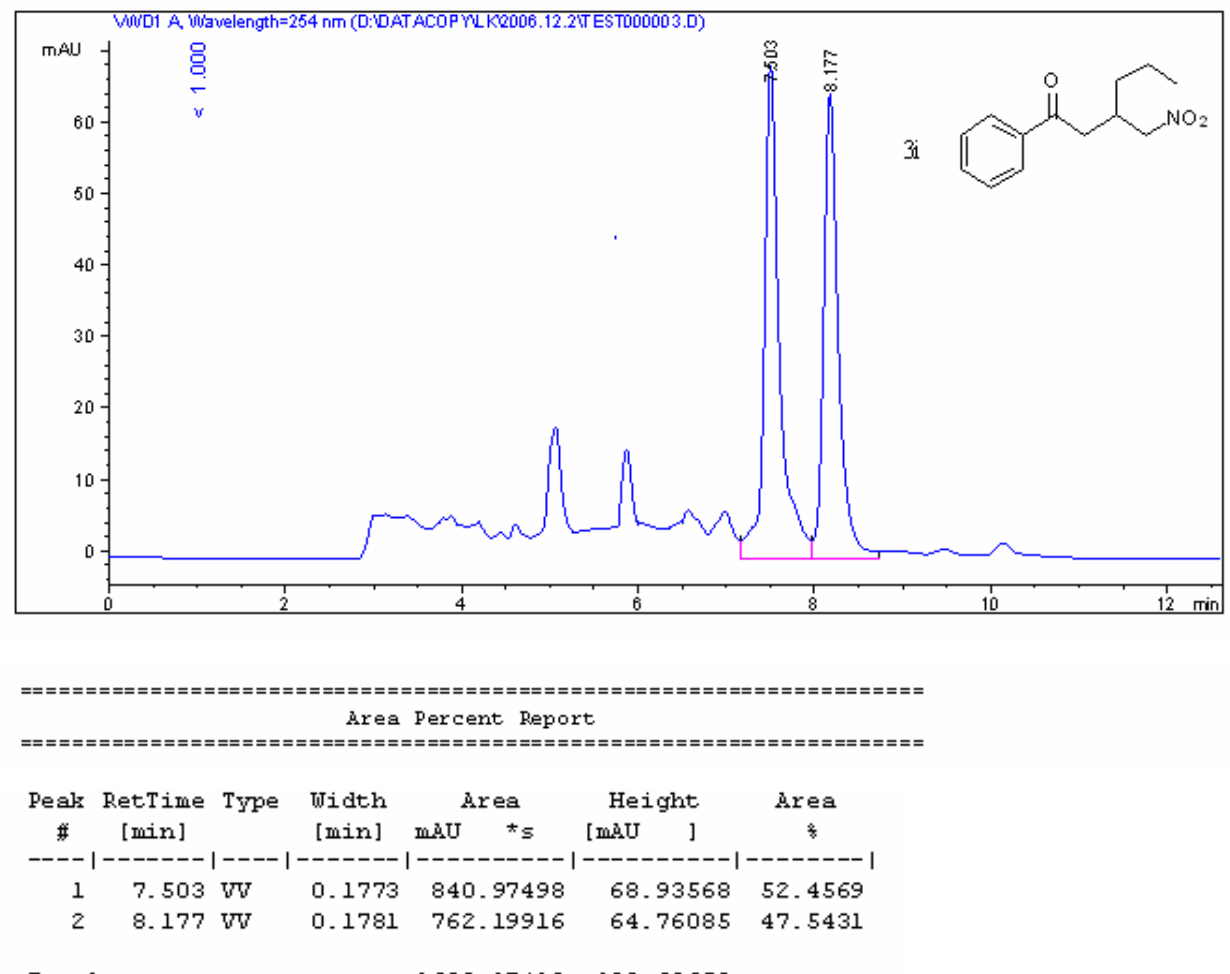

Totals :

$1603.17413 \quad 133.69653$

Sample Info : $254 \mathrm{~mm}, \mathrm{i}-\mathrm{Pr} 0 \mathrm{H}:$ hexane $=10^{-}: 90, \mathrm{AD}-\mathrm{H}, 1.0 \mathrm{ml} / \mathrm{min}$

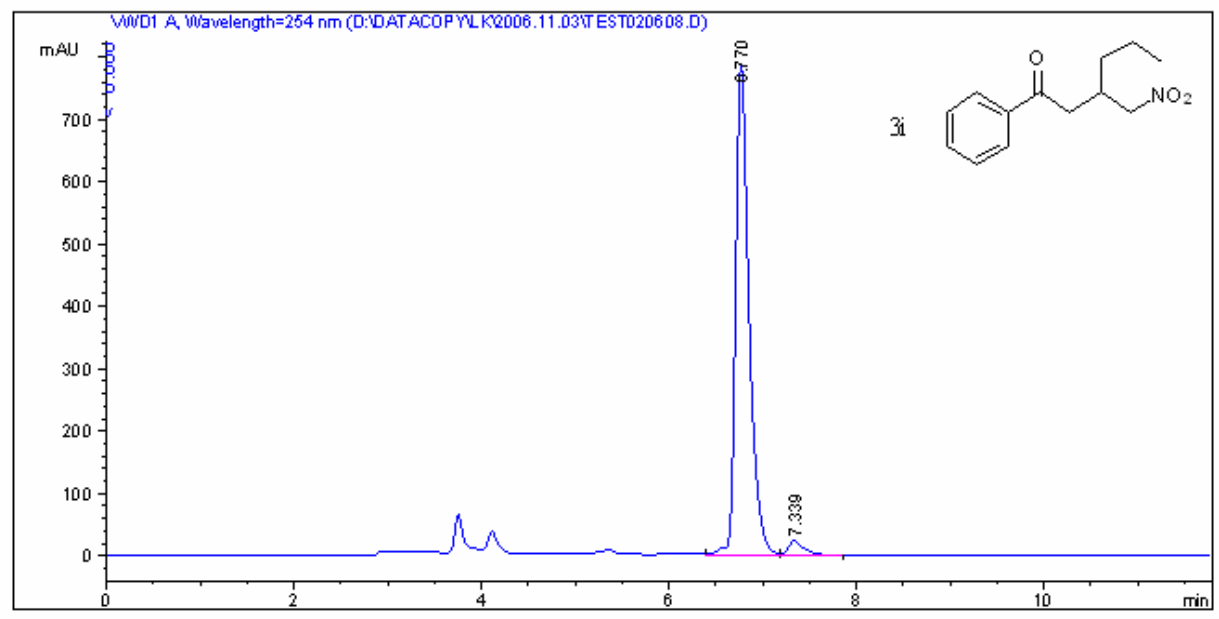

Area Percent Report

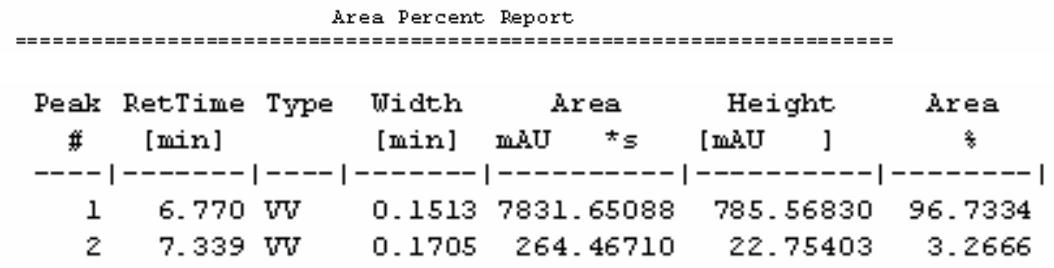

Totals :

$8096.11798 \quad 808.32233$ 


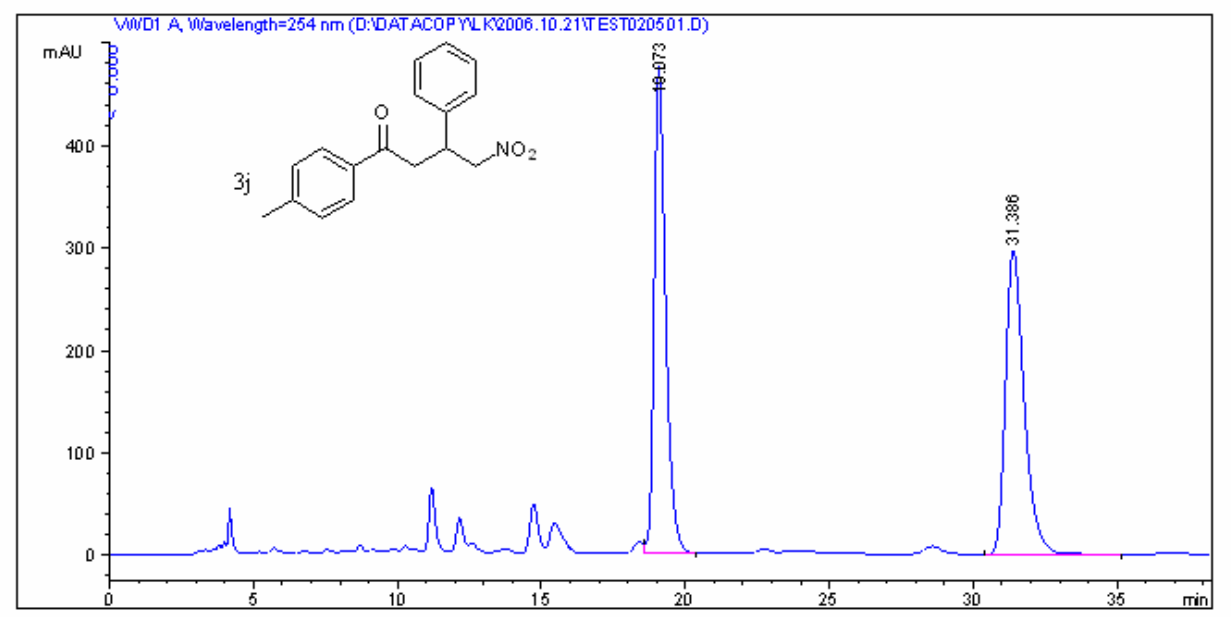

Area Percent Report

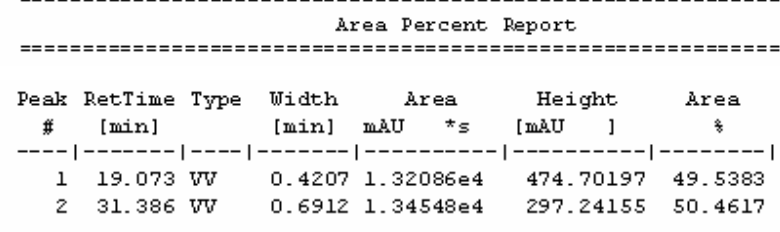

Totals : $\quad 2.66633 e 4 \quad 771.94351$

Sample Info : $254 \mathrm{~nm}, \mathrm{i}-\mathrm{Pr} 0 \mathrm{H}:$ hexane $=10: 90, \mathrm{AD}-\mathrm{H}, 1.0 \mathrm{ml} / \mathrm{min}$

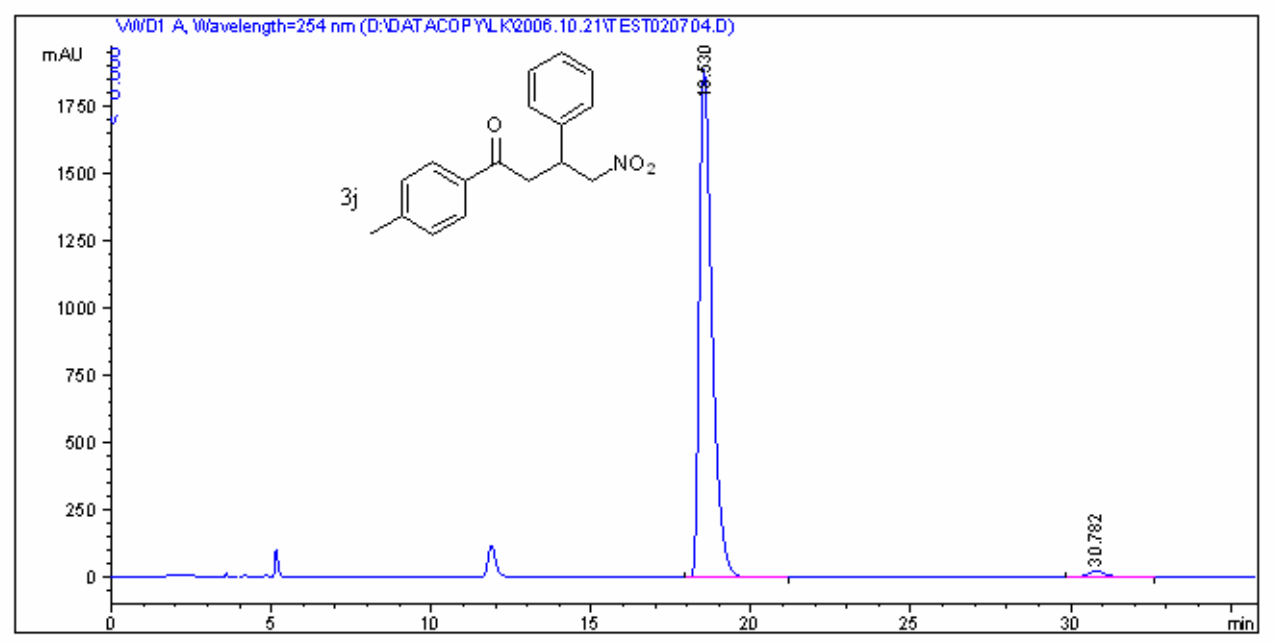

Area Percent Report

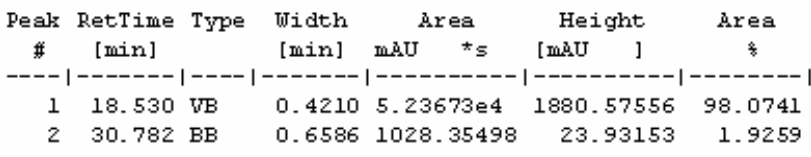

Totals :

$5.33956 \mathrm{e} \quad 1904.50709$ 


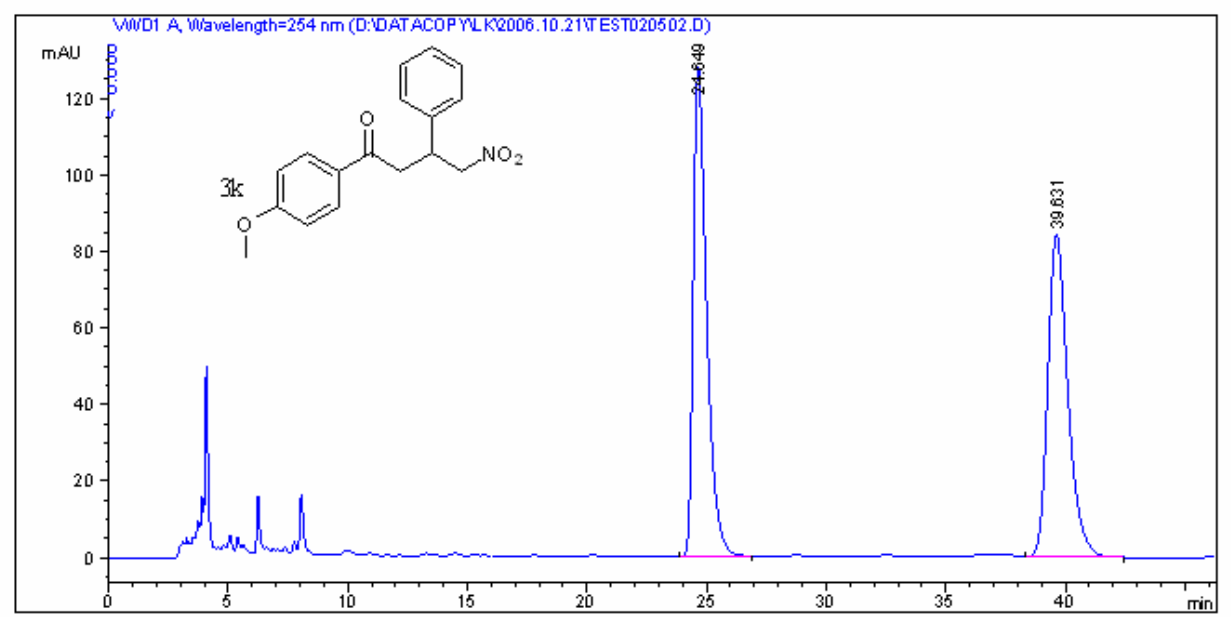

Area Percent Report

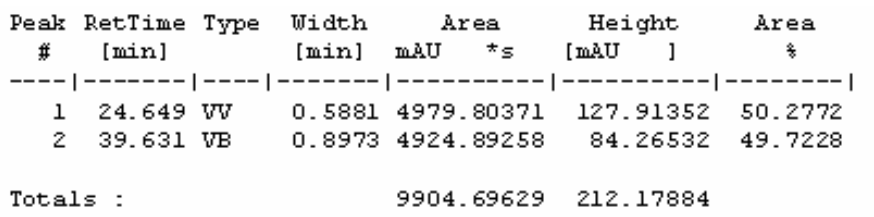

Sample Info : $254 \mathrm{~nm}, \mathrm{i}-\mathrm{PrOH}:$ hexane $=15: 85, \mathrm{AD}-\mathrm{H}, 1.0 \mathrm{ml} / \mathrm{min}$

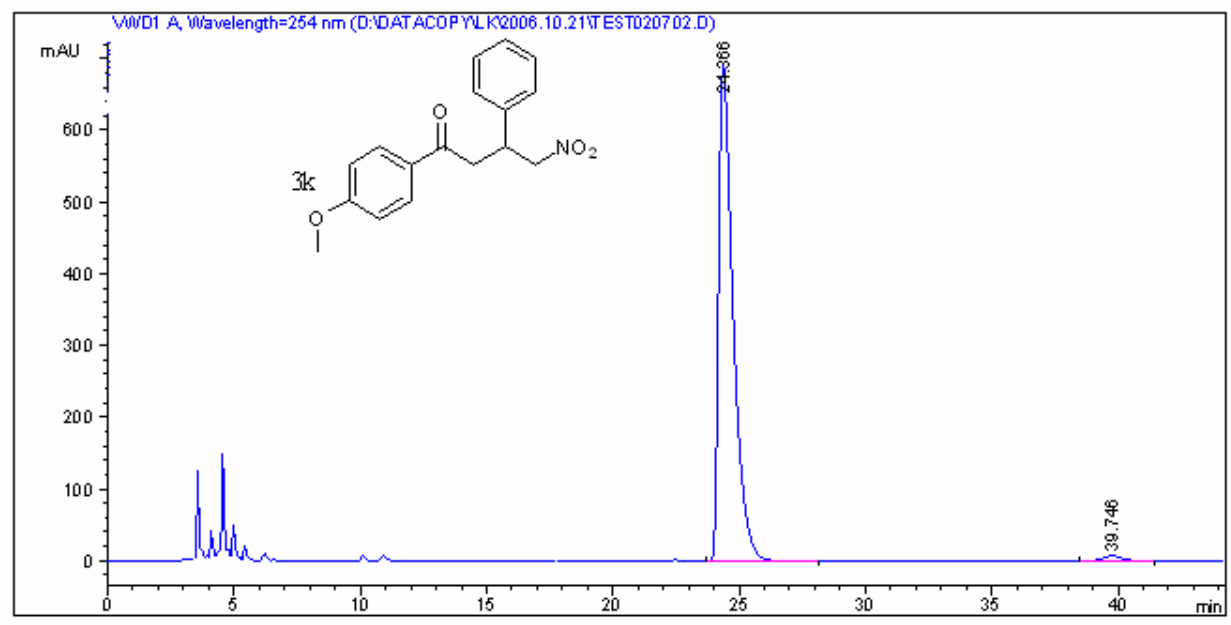

Area Percent Report

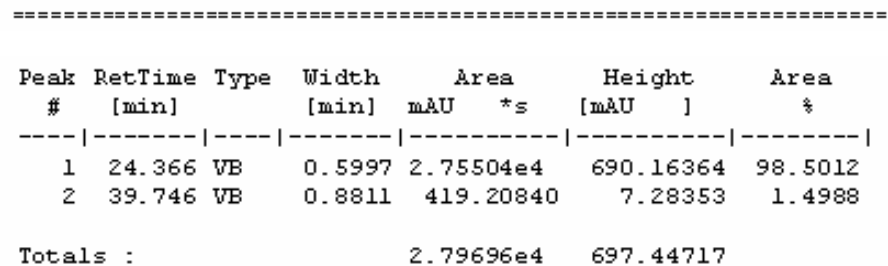

Totals :

2. $79696 \mathrm{e} 4697.44717$ 

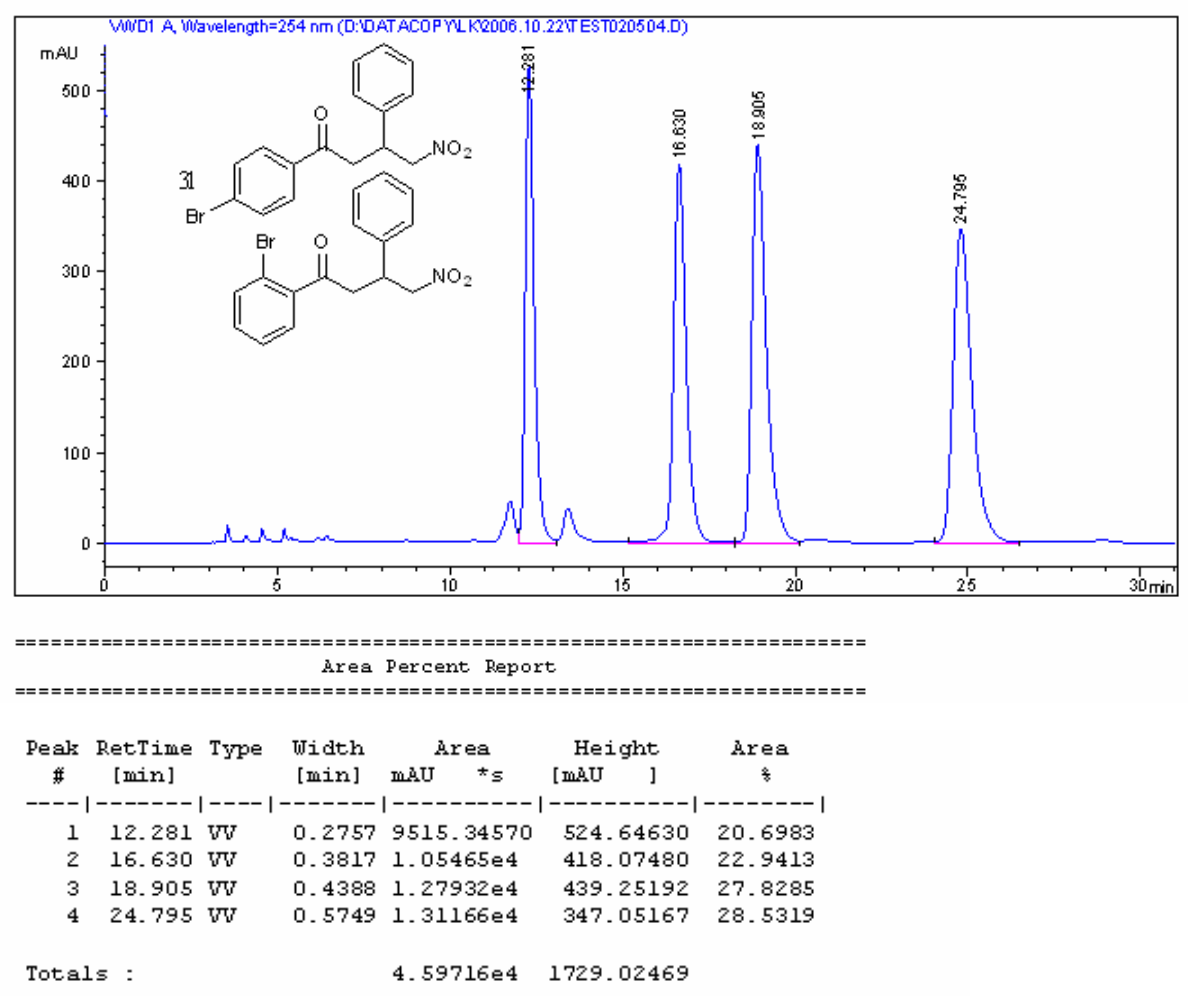

Sample Info : 254mm,i-proH: hexane $=15: 85, \mathrm{AD}-\mathrm{H}, 1.0 \mathrm{ml} / \mathrm{min}$

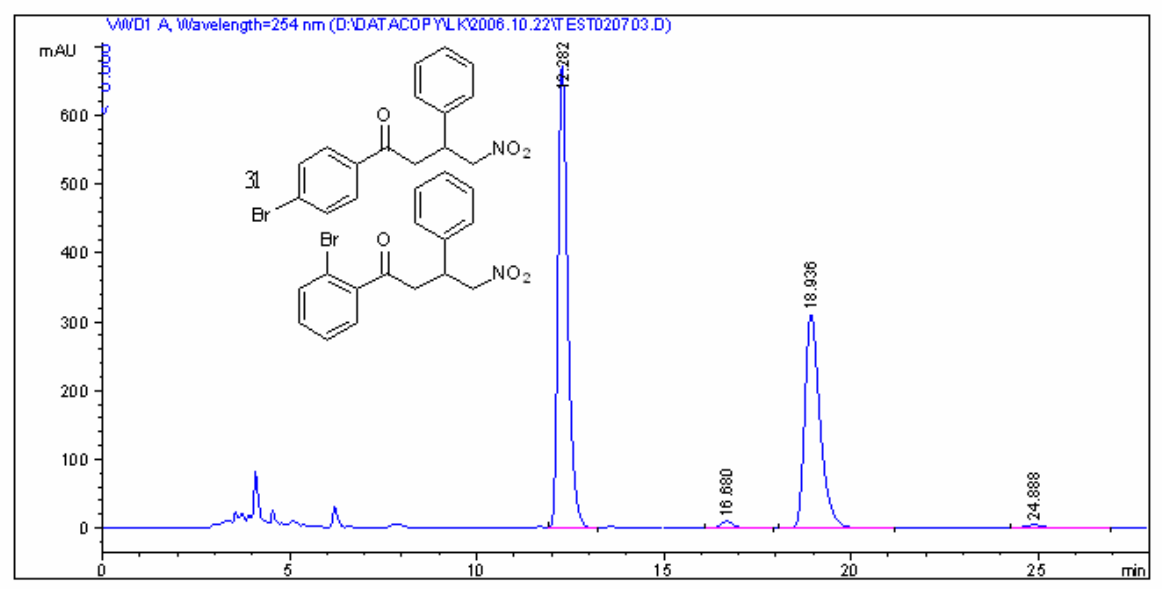

Area Percent Report

\begin{tabular}{|c|c|c|c|c|c|c|}
\hline Peat & RetTime & Type & Width & Àrea & Height & Area \\
\hline$\#$ & [min] & & [min] & InAU & [msU] & s \\
\hline & & & & 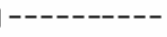 & ----------- & $-\cdots$ \\
\hline 1 & 12.282 & VV & 0.2782 & 1. $21790 \mathrm{e} 4$ & 672.72821 & 56.5573 \\
\hline 2 & 16.680 & VB & 0.3630 & 255.66861 & 10.70726 & 1. 1873 \\
\hline 3 & 18.936 & BV & 0.4385 & 8886.56152 & 310.73959 & 41.2678 \\
\hline 4 & 24.888 & VB & 0.6162 & 212.66685 & 5.08309 & 0.9876 \\
\hline ot & : & & & 2. $15339 \mathrm{e} 4$ & 999.25815 & \\
\hline
\end{tabular}




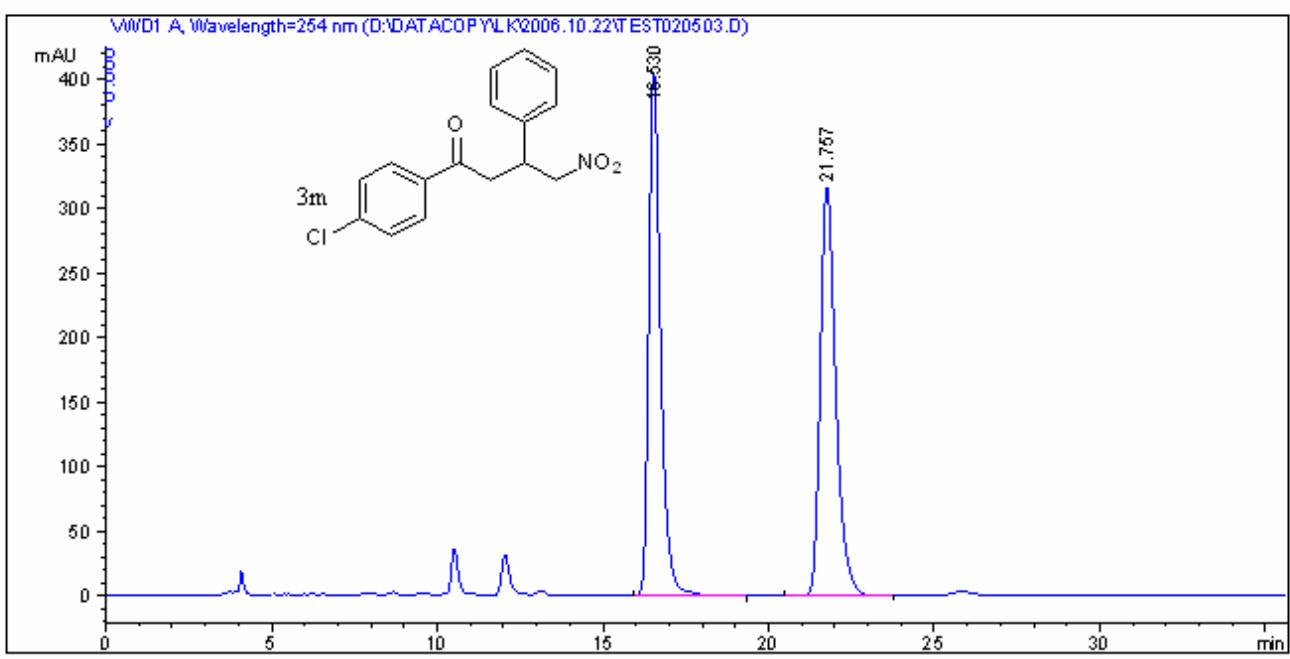

Area Percent Report

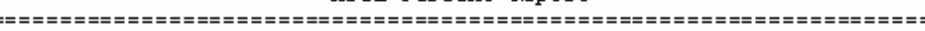

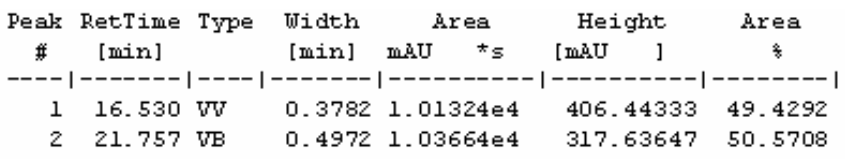

Totals :

$2.04988 e 4 \quad 724.07980$

Sample Info : $254 \mathrm{~mm}, \mathrm{i}-\mathrm{Pr} 0 \mathrm{H}:$ hexane $=15: 85, \mathrm{AD}-\mathrm{H}, 1.0 \mathrm{ml} / \mathrm{min}$

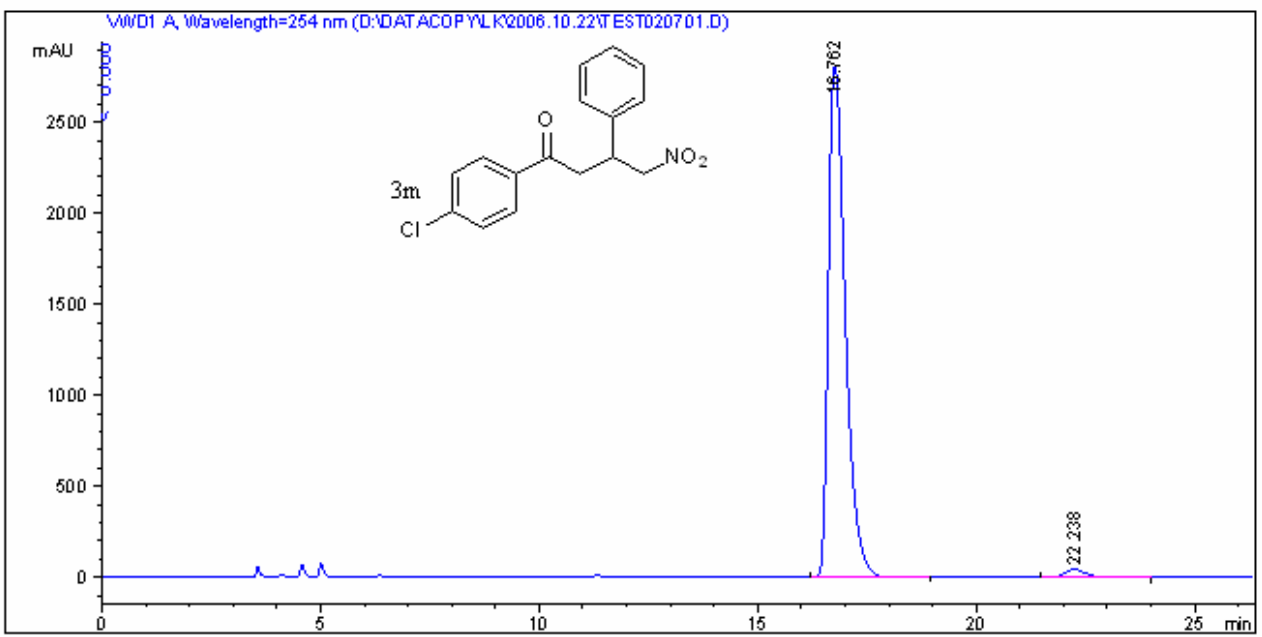

Area Percent Report

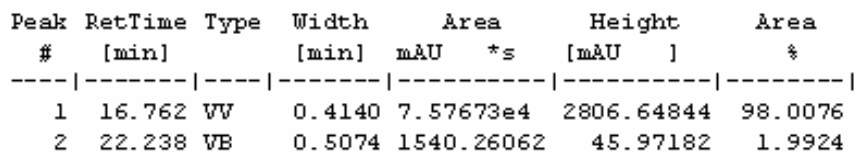

Totals :

$7.73076 \mathrm{e} 42852.62025$ 
Sample Info : 254nm,i-ProH: hexane $=10: 90, \mathrm{AD}-\mathrm{H}, 1.0 \mathrm{ml} / \mathrm{min}$

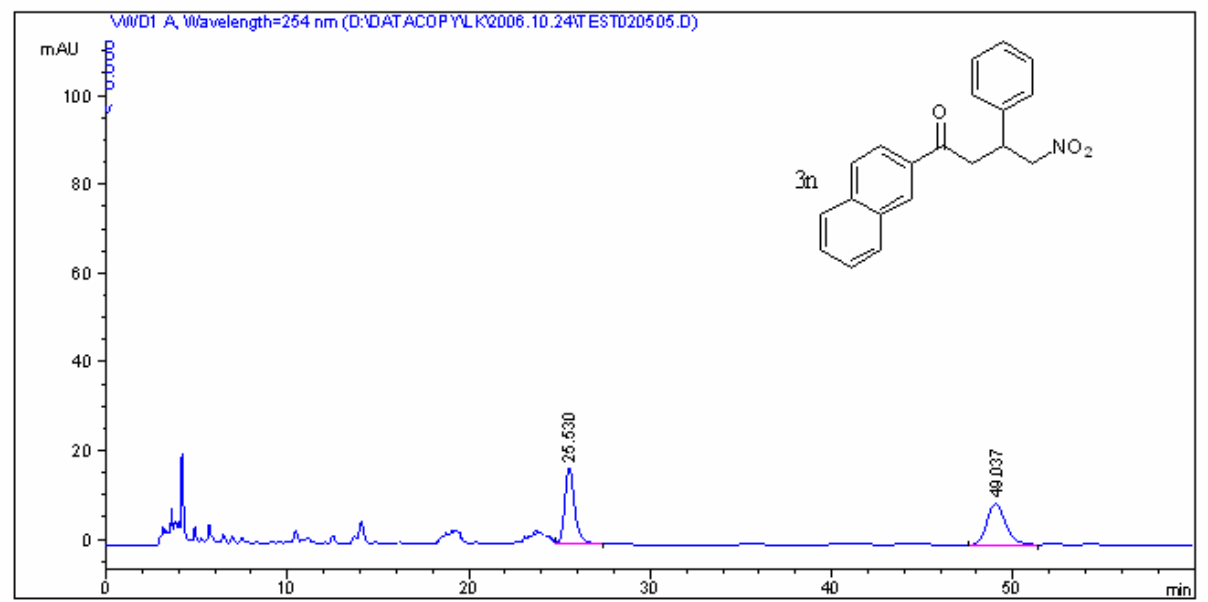

Area Percent Report

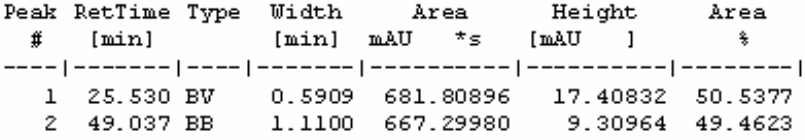

Totals :

$1349.10876 \quad 26.71795$

Sample Info : $254 \mathrm{~mm}, \mathrm{i}-\mathrm{PrOH}:$ hexane $=10^{-} 90, \mathrm{AD}-\mathrm{H}, 1.0 \mathrm{ml} / \mathrm{min}$

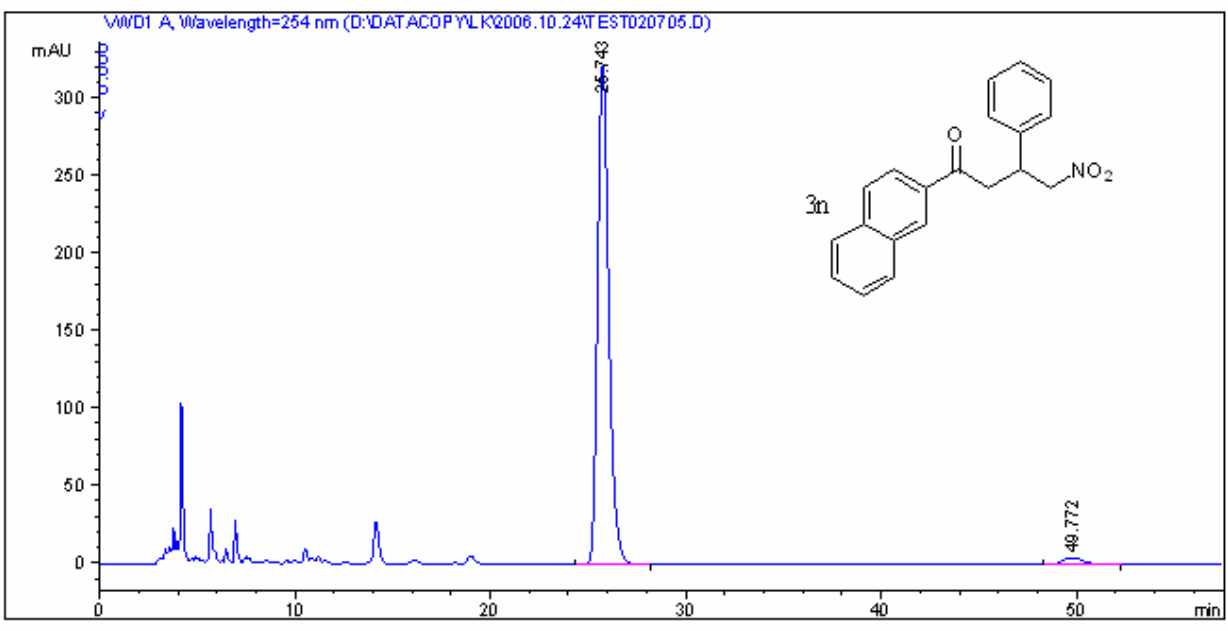

Area Percent Report

\begin{tabular}{|c|c|c|c|c|c|c|}
\hline \multirow{2}{*}{$\begin{array}{c}\text { Peak } \\
\#\end{array}$} & \multirow{2}{*}{$\begin{array}{l}\text { RetTime } \\
\text { [min] }\end{array}$} & \multirow[t]{2}{*}{ Type } & \multirow{2}{*}{$\begin{array}{l}\text { Width } \\
\text { [min] }\end{array}$} & Area & Height & \multirow{2}{*}{$\begin{array}{c}\text { Area } \\
8\end{array}$} \\
\hline & & & & $\mathrm{mAU}$ & {$[\mathrm{m} \mathbf{A} U$} & \\
\hline----1 & $\mid------$ & $\mid---$ & ------ & |---------- & ----------1 & -------1 \\
\hline 1 & 25.743 & VB & 0.6117 & $1.28484 \mathrm{e} 4$ & 321.60965 & 97.3742 \\
\hline 2 & 49.772 & $\mathrm{BB}$ & 1.1182 & 346.47461 & 4.62702 & 2.6258 \\
\hline
\end{tabular}

Totals :

1.31949 e4 326.23667 

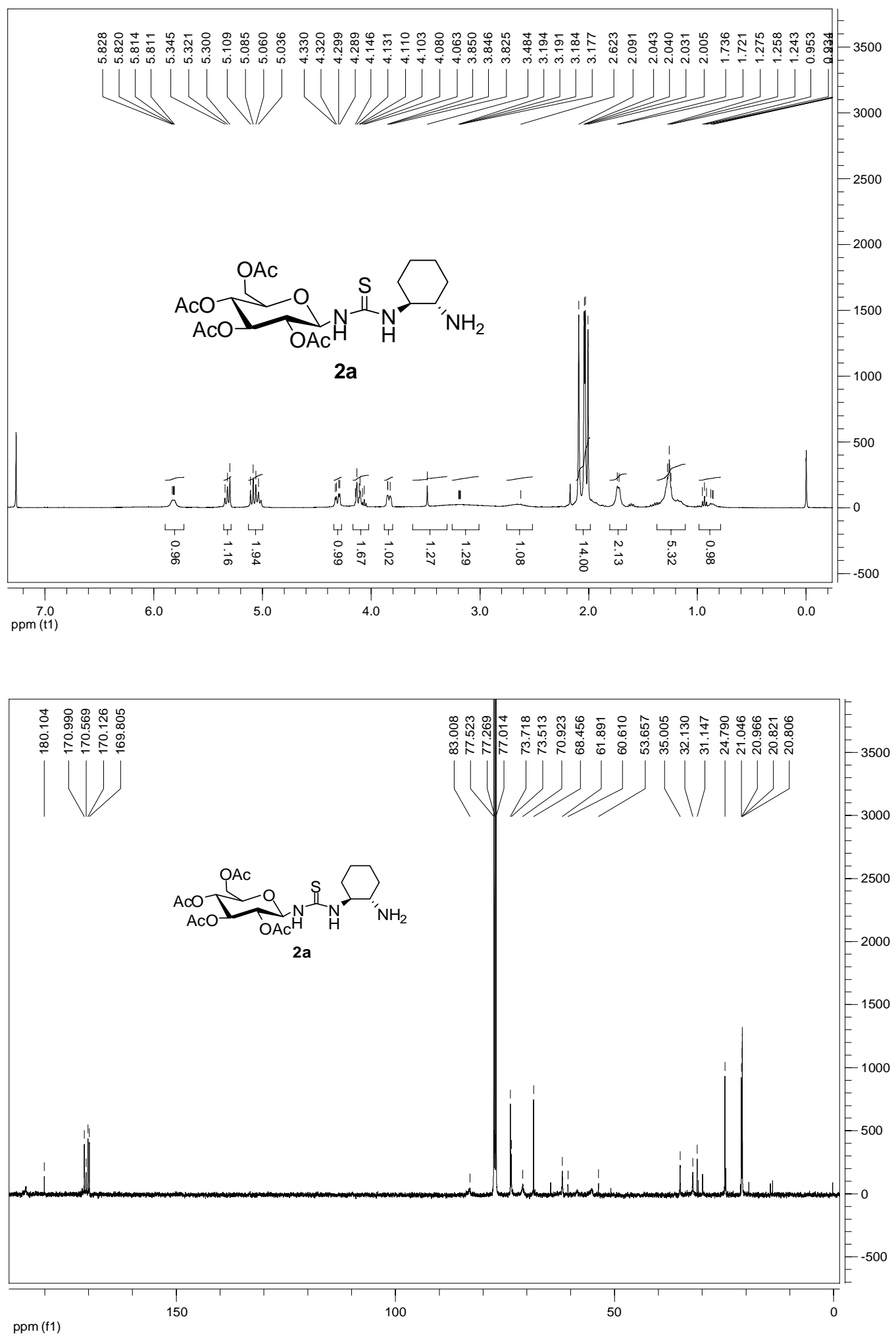

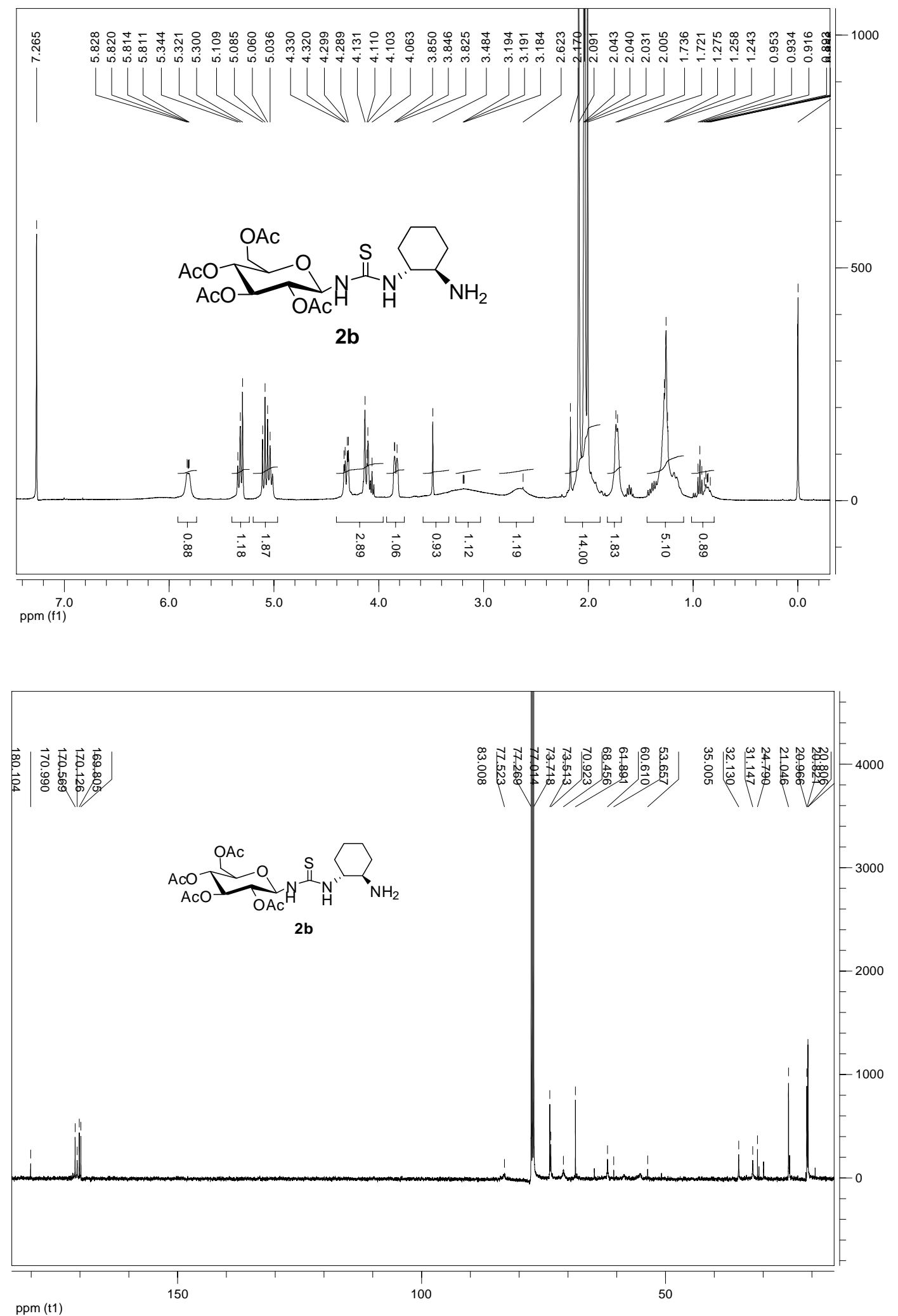

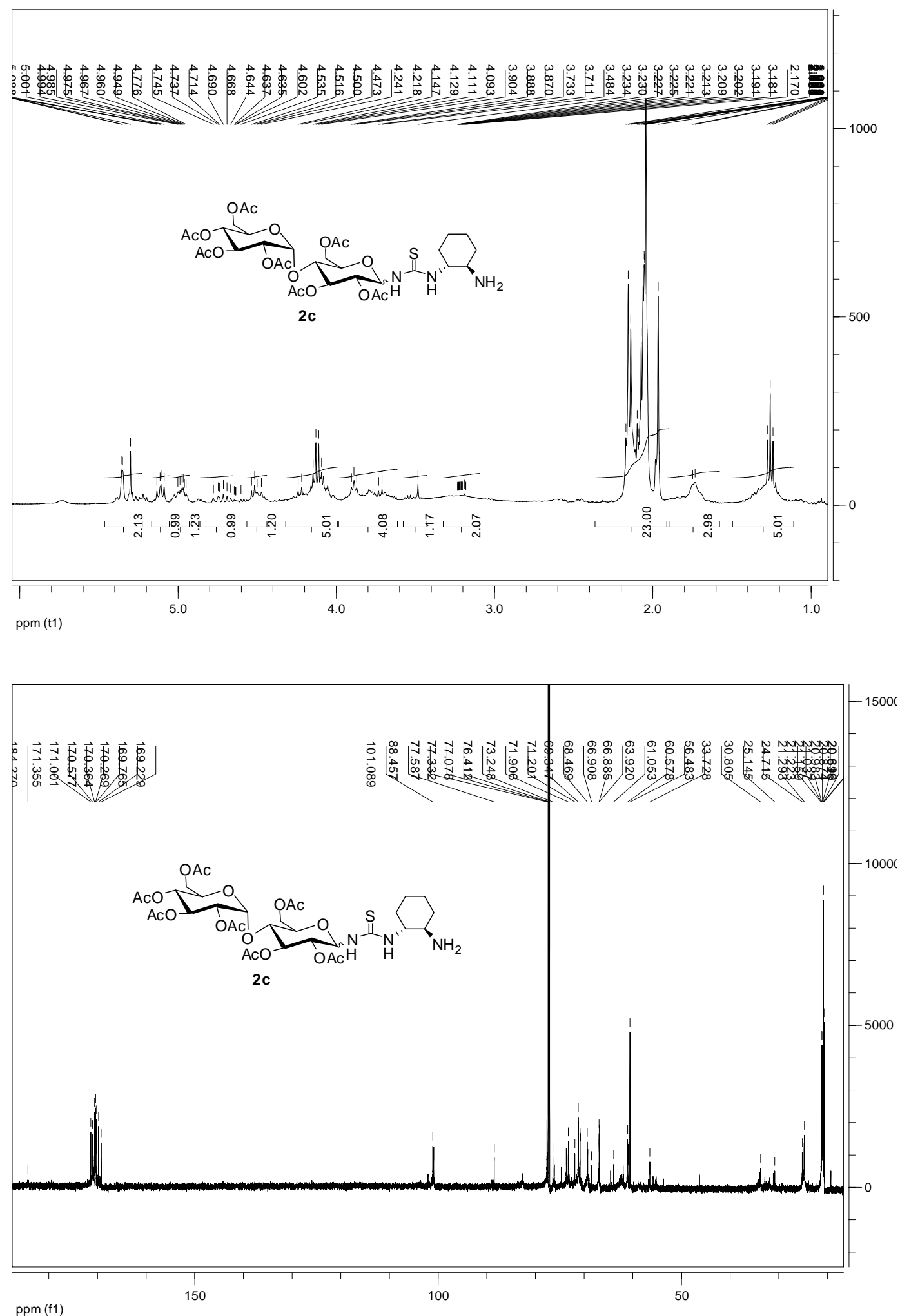

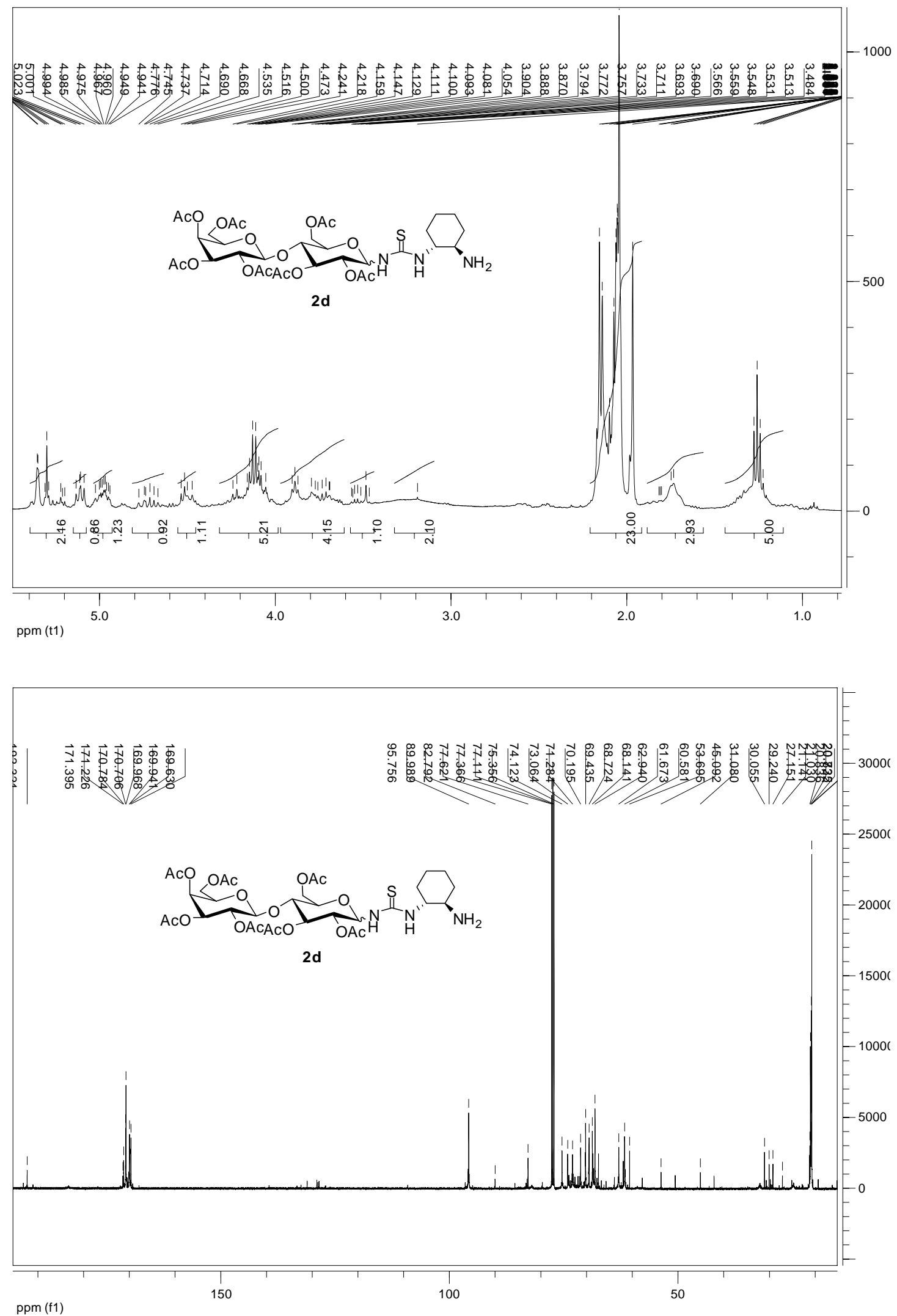

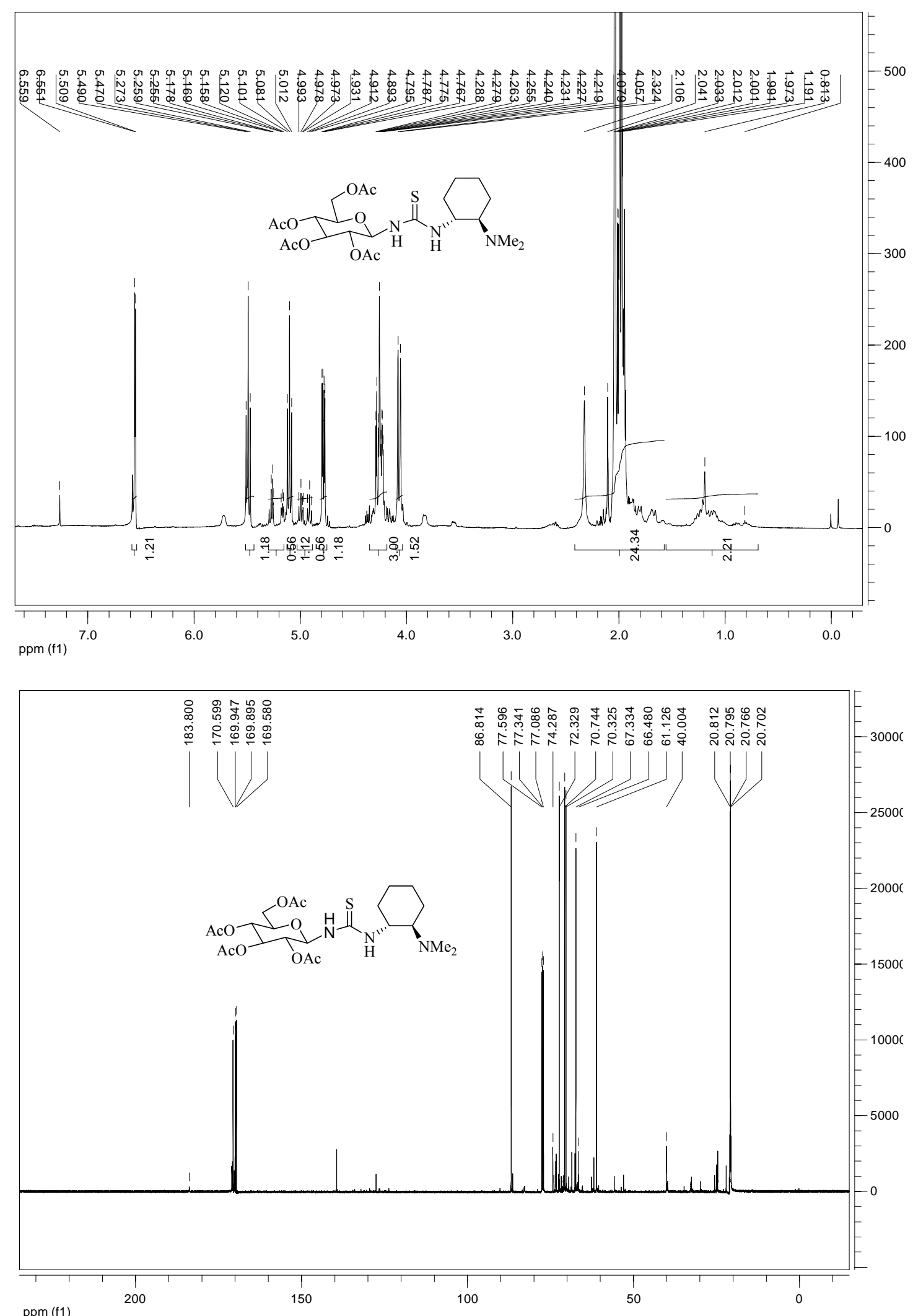


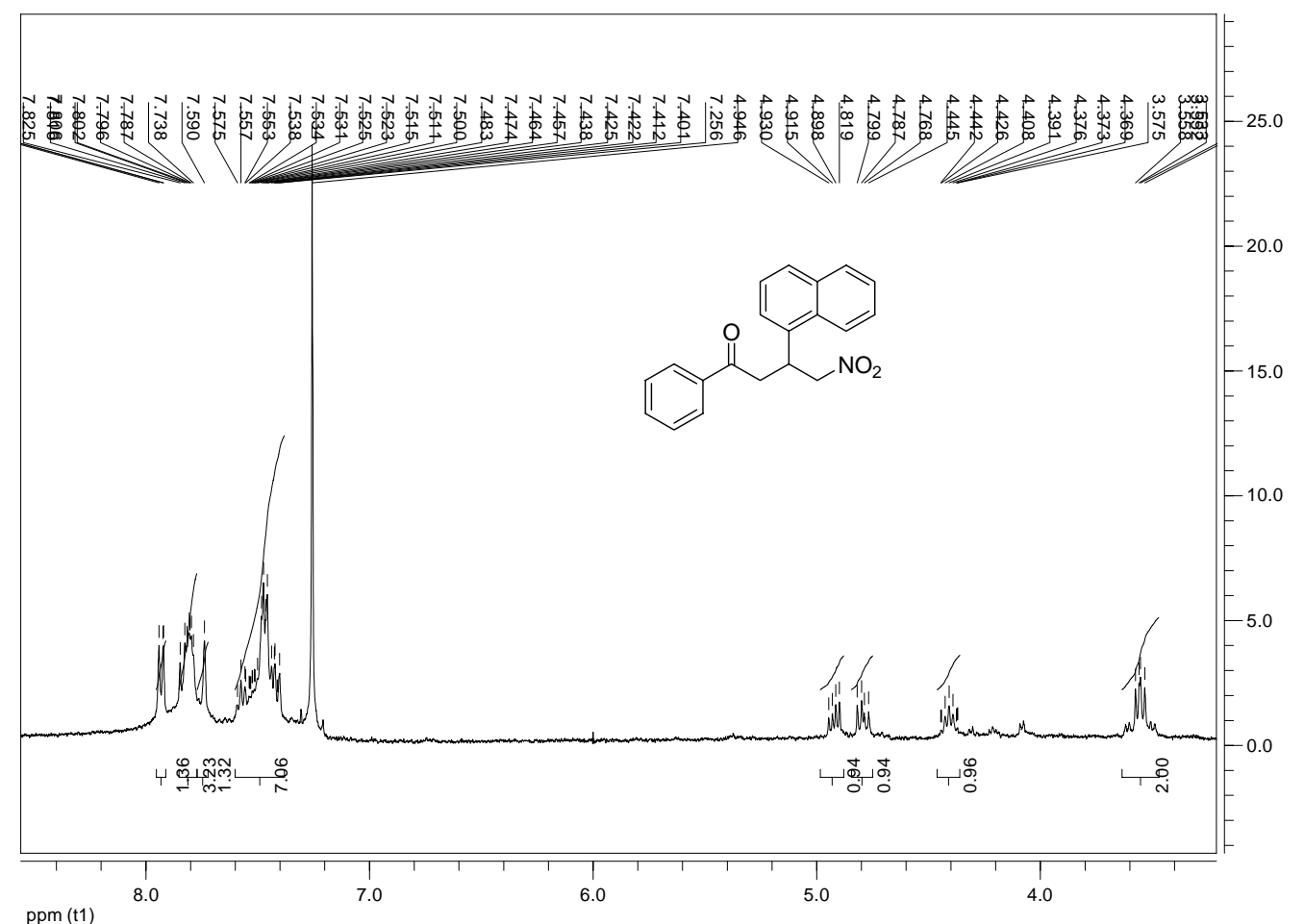

ppm (t1)

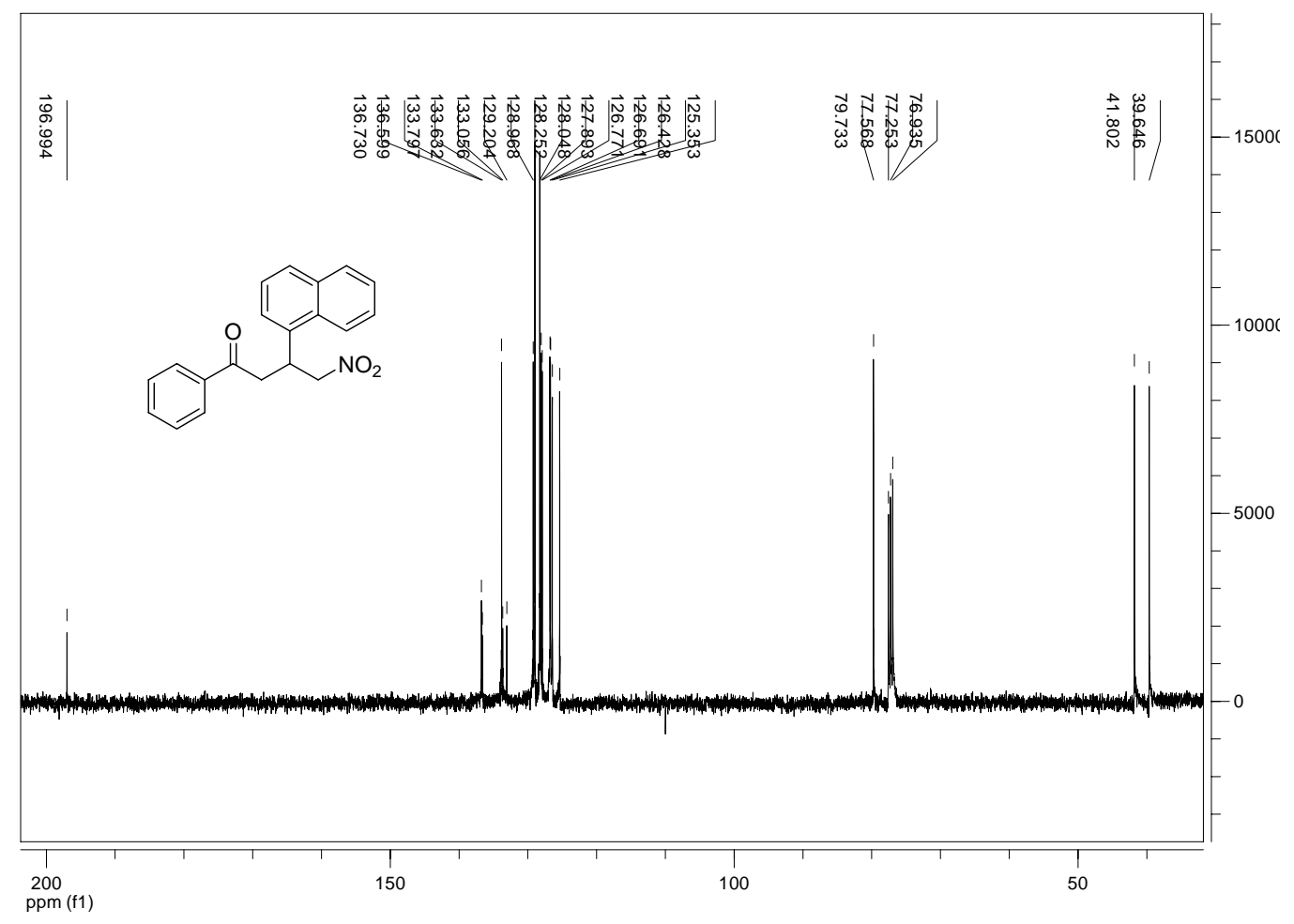




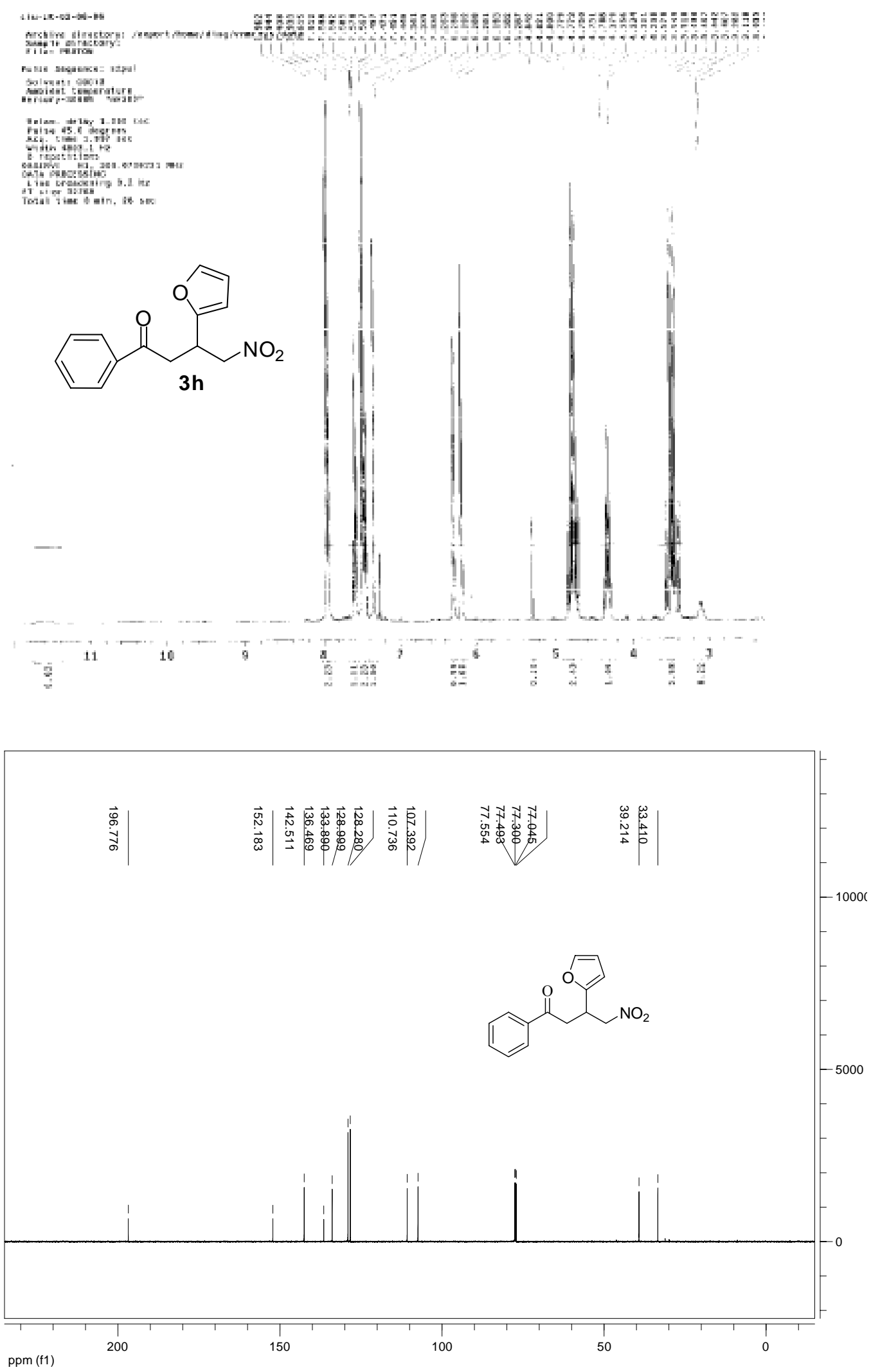



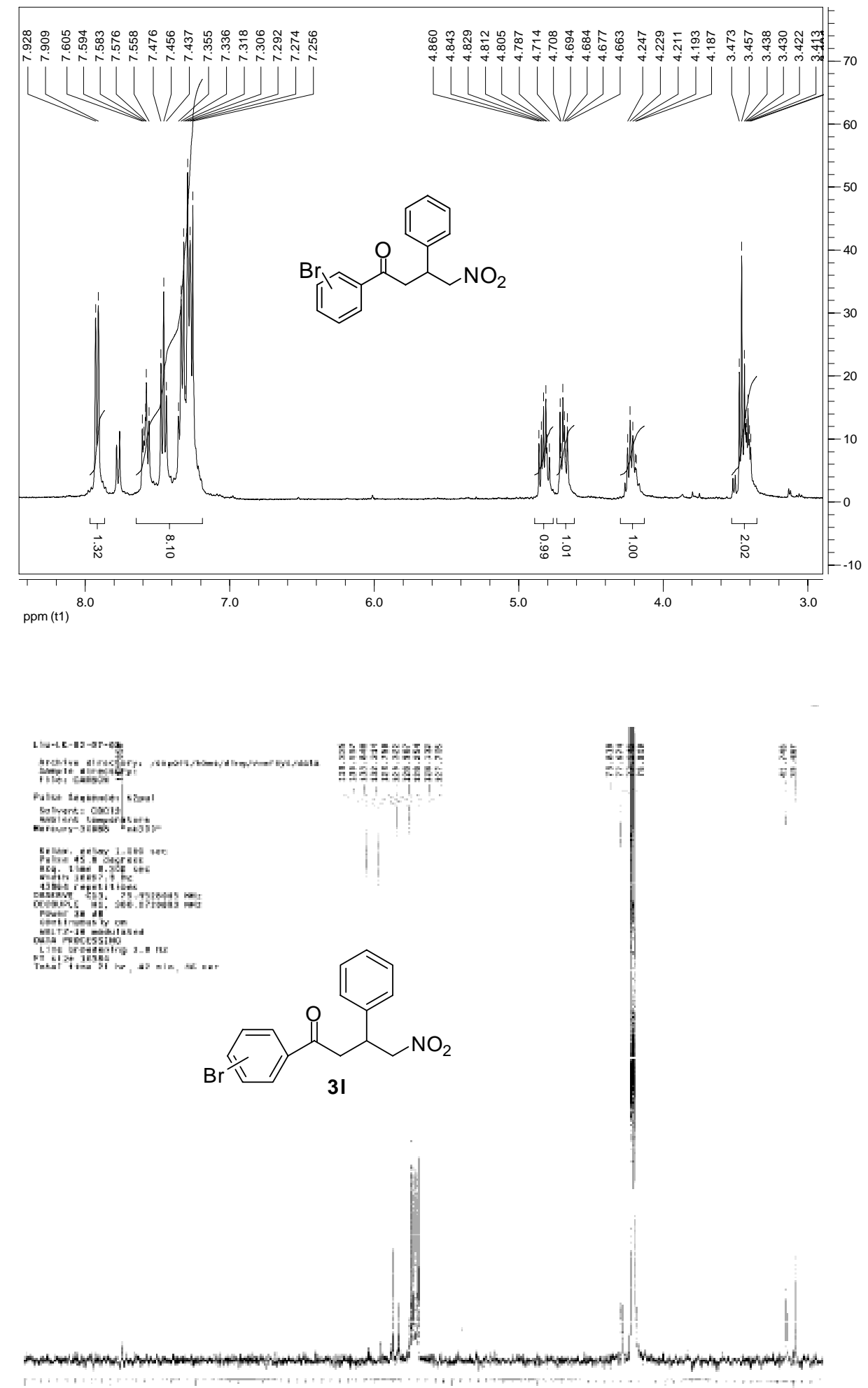

216

$160 \quad$ Na

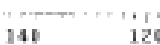

aด

60 

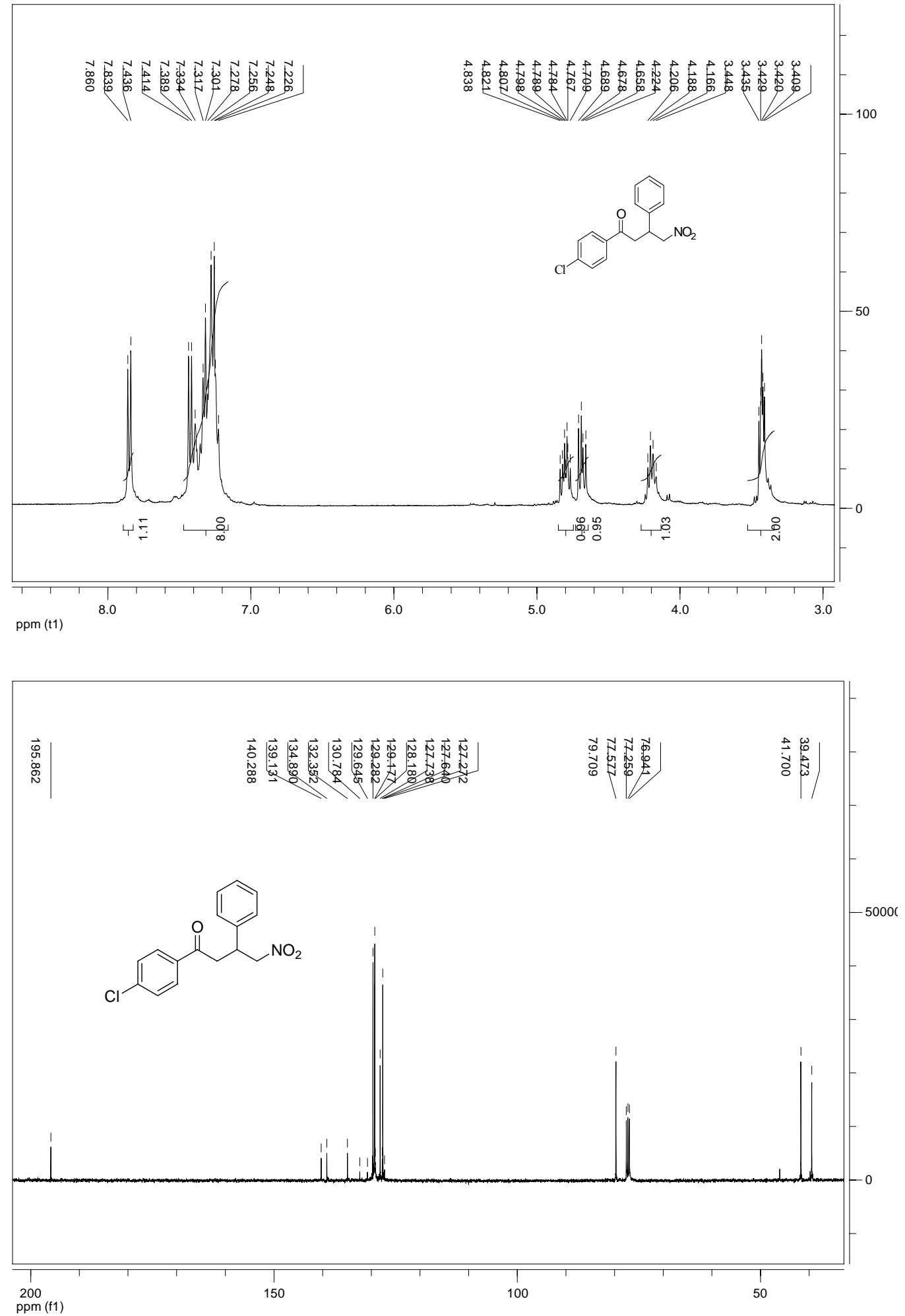